\title{
Neuronal L-Type Calcium Channel Signaling to the Nucleus Requires a Novel CaMKII $\alpha$-Shank3 Interaction
}

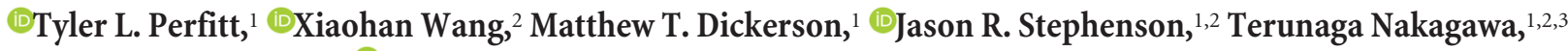 \\ David A. Jacobson, ${ }^{1}$ and ${ }^{D}$ Roger J. Colbran ${ }^{1,2,4}$ \\ ${ }^{1}$ Department of Molecular Physiology and Biophysics, ${ }^{2}$ Vanderbilt Brain Institute, ${ }^{3}$ Center for Structural Biology, and ${ }^{4}$ Vanderbilt-Kennedy Center for \\ Research on Human Development, Vanderbilt University School of Medicine, Nashville, Tennessee 37232-0615
}

The activation of neuronal plasma membrane $\mathrm{Ca}^{2+}$ channels stimulates many intracellular responses. Scaffolding proteins can preferentially couple specific $\mathrm{Ca}^{2+}$ channels to distinct downstream outputs, such as increased gene expression, but the molecular mechanisms that underlie the exquisite specificity of these signaling pathways are incompletely understood. Here, we show that complexes containing CaMKII and Shank3, a postsynaptic scaffolding protein known to interact with L-type calcium channels (LTCCs), can be specifically coimmunoprecipitated from mouse forebrain extracts. Activated purified CaMKII $\alpha$ also directly binds Shank 3 between residues 829 and 1130. Mutation of Shank3 residues ${ }^{949}$ Arg-Arg-Lys ${ }^{951}$ to three alanines disrupts CaMKII binding in vitro and CaMKII association with Shank3 in heterologous cells. Our shRNA/rescue studies revealed that Shank3 binding to both CaMKII and LTCCs is important for increased phosphorylation of the nuclear CREB transcription factor and expression of c-Fos induced by depolarization of cultured hippocampal neurons. Thus, this novel CaMKII-Shank3 interaction is essential for the initiation of a specific long-range signal from LTCCs in the plasma membrane to the nucleus that is required for activity-dependent changes in neuronal gene expression during learning and memory.

Key words: calcium; CaMKII; ion channel; scaffolding protein; transcriptional regulation

\section{Significance Statement}

Precise neuronal expression of genes is essential for normal brain function. Proteins involved in signaling pathways that underlie activity-dependent gene expression, such as CaMKII, Shank3, and L-type calcium channels, are often mutated in multiple neuropsychiatric disorders. Shank3 and CaMKII were previously shown to bind L-type calcium channels, and we show here that Shank3 also binds to CaMKII. Our data show that each of these interactions is required for depolarization-induced phosphorylation of the CREB nuclear transcription factor, which stimulates the expression of c-Fos, a neuronal immediate early gene with key roles in synaptic plasticity, brain development, and behavior.

\section{Introduction}

Neuronal depolarization stimulates $\mathrm{Ca}^{2+}$ influx and multiple intracellular signaling pathways that are essential for normal brain

Received April 19, 2019; revised Jan. 23, 2020; accepted Jan. 28, 2020.

Author contributions: T.L.P., X.W., and R.J.C. designed research; T.L.P. and M.T.D. performed research; T.L.P., X.W., J.R.S., T.N., and D.A.J. contributed unpublished reagents/analytic tools; T.L.P. and R.J.C. analyzed data; T.L.P. wrote the first draft of the paper; T.L.P., X.W., M.T.D., J.R.S., T.N., D.A.J., and R.J.C. edited the paper; T.L.P. and R.J.C. wrote the paper.

This work was supported by the National Institutes of Health Grants T32-DK007563 to T.L.P., T32-MH065215 to J.R.S., R01-HD061543 to T.N., R01-DK067392 and R01-DK115620 to D.A.J., and R01-MH063232 and R01-NS078291 to R.J.C.; and American Heart Association Grants 14PRE18420020 to X.W. and 18PRE33960034 to T.L.P. The content is solely the responsibility of the authors and does not necessarily represent the official views of the National Institutes of Health. Confocal imaging and analysis were performed in part through the use of the Vanderbilt Cell Imaging Shared Resource (supported by National Institutes of Health Grants CA68485, DK20593, DK58404, DK59637, and EY08126). We thank Drs. Craig Garner, Luk Van Parijs, Diane Lipscombe, and Winship Herr for generously providing various plasmids (detailed in Materials and Methods).

The authors declare no competing financial interests.

Correspondence should be addressed to Roger J.Colbran at roger.colbran@vanderbilt.edu. functions, including excitation-transcription (E-T) coupling. $\mathrm{Ca}^{2+}$-dependent phosphorylation of the nuclear transcription factor CREB at Ser ${ }^{133}$ stimulates the transcription of immediate early genes encoding multiple proteins (e.g., c-Fos, BDNF, homerla) that play key roles in learning and memory (Dolmetsch, 2003; Flavell and Greenberg, 2008; Benito et al., 2011; Bading, 2013). Disruptions in activity-dependent gene expression are associated with multiple neuropsychiatric disorders (Ebert and Greenberg, 2013; Gallo et al., 2018) that have been linked to mutations in $\mathrm{Ca}^{2+}$ signaling proteins, including L-type calcium channels (LTCCs) and CaMKII (Nyegaard et al., 2010; Pinggera et al., 2015, 2017; Dick et al., 2016; Limpitikul et al., 2016; Pinggera and Striessnig, 2016; Küry et al., 2017; Stephenson et al., 2017; Akita et al., 2018; Chia et al., 2018; Cohen et al., 2018; Moon et al., 2018; 
Proietti Onori et al., 2018). For example, Timothy syndrome is caused by mutations in the $\mathrm{Ca}_{\mathrm{V}} 1.2$ LTCC $\alpha 1$ subunit that can disrupt neuronal E-T coupling (Li et al., 2016), contributing to neurobehavioral symptoms of this complex disorder, including autism spectrum disorder (ASD). Recent studies have shown that initiation of LTCC-dependent E-T coupling requires recruitment of multiple CaMKII holoenzymes to a nanodomain close to LTCCs (Wheeler et al., 2008; Ma et al., 2014; Wang et al., 2017), and that E-T coupling is disrupted by a CaMKII mutation linked to intellectual disability (Cohen et al., 2018). However, molecular mechanisms underlying the organization and function of this LTCC nanodomain remain incompletely understood.

CaMKII has critical roles in neuronal signaling and plasticity. $\mathrm{Ca}^{2+} /$ calmodulin (CaM) binding to 12-subunit CaMKII holoenzymes stimulates intersubunit autophosphorylation at Thr286 (in CaMKII $\alpha$ ), a key mechanism underlying learning and memory (for review, see Lisman et al., 2012; Hell, 2014; Shonesy et al., 2014). Thr286-autophosphorylated CaMKII can remain autonomously active after the initial $\mathrm{Ca}^{2+}$ influx dissipates and $\mathrm{Ca}^{2+}$ / CaM dissociates (Lai et al., 1986; Miller and Kennedy, 1986; Miller et al., 1988), leading to sustained phosphorylation of downstream targets. Activated CaMKII also interacts with a number of other synaptic proteins. For example, activated CaMKII binding to NMDAR GluN2B subunits is important for CaMKII targeting to dendritic spines and for normal synaptic plasticity (Strack and Colbran, 1998; Bayer et al., 2001, 2006; Halt et al., 2012). In addition, densin-180 can bind to both $\mathrm{Ca}_{\mathrm{V}} 1.3$ LTCCs and CaMKII, thereby suppressing $\mathrm{Ca}^{2+}$-dependent inactivation of $\mathrm{Ca}_{\mathrm{V}} 1.3$ and facilitating overall $\mathrm{Ca}^{2+}$ entry via LTCCs (Jenkins et al., 2010; Jiao et al., 2011). A recent study also showed that CaMKII binding to mGlu5 metabotropic glutamate receptors modulates the mobilization of intracellular $\mathrm{Ca}^{2+}$ stores (Marks et al., 2018). This emerging evidence supports the hypothesis that direct interactions of CaMKII with CaMKII-associated proteins (CaMKAPs) are important for synaptic signaling.

Our recent proteomics study identified Shank3 as one of the most abundant proteins in CaMKII complexes isolated from a Triton X-100/deoxycholate-solubilized synapse-enriched subcellular fraction (Baucum et al., 2015). Canonically, Shank3 contains an N-terminal ankyrin repeat domain, SH3 and PDZ domains, a proline-rich region with binding sites for homer and cortactin, and a C-terminal SAM domain that mediates oligomerization (Naisbitt et al., 1999). Diverse mutations in Shank3 are strongly linked to ASD and schizophrenia (Leblond et al., 2014; Soler et al., 2018), while a chromosomal deletion causes haploinsufficiency of the SHANK3 gene in 22q13 deletion syndrome (Phelan-McDermid syndrome), another neurodevelopmental disorder associated with ASD (Harony-Nicolas et al., 2015). Indeed, knockdown of Shank3 expression in cultured hippocampal neurons reduces dendritic spine formation and mEPSC frequency (Verpelli et al., 2011), and several Shank3 mutant mouse lines display different combinations of deficits in synaptic transmission, social behavior, and learning (for review, see Monteiro and Feng, 2017). The Shank3 PDZ domain can bind to a C-terminal PDZ binding motif in $\mathrm{Ca}_{\mathrm{V}} 1.3$ LTCCs, and deletion of this PDZ binding motif disrupts $\mathrm{Ca}_{\mathrm{V}} 1.3$ clustering in neuronal dendrites and LTCC-dependent E-T coupling (Zhang et al., 2005). Therefore, we hypothesized that direct interactions of Shank3 with LTCCs and CaMKII are important for CaMKII function within the LTCC nanodomain that is required for neuronal E-T coupling.
Here we identify a novel binding site for CaMKII in Shank3 and show that CaMKII activation, either by $\mathrm{Ca}^{2+} / \mathrm{CaM}$ binding or Thr286 autophosphorylation, is required for this interaction. Using site-directed mutagenesis, we identified three residues in Shank3 that are critical for this interaction. Mutation of these residues in full-length Shank3 disrupts coimmunoprecipitation and colocalization with CaMKII. In addition, this Shank3 mutation disrupts LTCC/CaMKII-dependent E-T coupling to CREB and subsequent c-Fos expression in hippocampal neurons.

\section{Materials and Methods}

Animals. All mice were housed on a $12 \mathrm{~h}$ light-dark cycle with food and water ad libitum. Camk2a-/- (referred to as CaMKII $\alpha$-KO) and Camk2 $a^{\text {tm2Sva }}$ (RRID:MGI:3764491, referred to as CaMKII $\alpha^{\text {T286A }}$ ) mice on a C57B/6J background were described previously (Giese et al., 1998; Marks et al., 2018). WT and homozygous littermates were generated using a HetxHet breeding strategy. Both male and female mice age P28P30 were used for biochemical studies. All animal experiments were approved by the Vanderbilt University Institutional Animal Care and Use Committee and were performed following the National Institutes of Health's Guide for the care and use of laboratory animals.

Antibodies used. The following antibodies were used for immunoblotting at the indicated dilutions: mouse anti-CaMKII $\alpha$ 6G9 (Thermo Fisher Scientific; catalog \#MA1-048, RRID:AB_325403, 1:5000), pT286 CaMKII $\alpha$ (Santa Cruz Biotechnology; catalog \#sc-12886-R, RRID: AB_2067915, 1:3000), mouse anti-Shank3 (University of California at Davis/National Institutes of Health NeuroMab Facility; catalog \#N367/ 62, RRID:AB_2315920, 1:2000), rabbit monoclonal anti-Shank3 (D5K6R) (Cell Signaling Technology; catalog \#64555, RRID:AB_2799661, 1:3000), goat anti-GST (GE Healthcare Life Sciences; catalog \#27-4577-01, 1:5000), polyclonal goat CaMKII antibody (RRID:AB_2631234, 1:5000) (McNeill and Colbran, 1995), mouse anti-PSD-95 (NeuroMab, catalog \#75-028, RRID:AB_2292909, 1:50,000), mouse anti-GFP (Vanderbilt Antibody and Protein Resource; catalog \#1C9A5, 1:3000), mouse anti-HA (BioLegend; catalog \#901503, RRID:AB_2565005, 1:3000), HRP-conjugated anti-rabbit (Promega; catalog \#W4011, RRID:AB_430833, 1:6000), HRP-conjugated anti-mouse (Promega, catalog \#W4021, RRID: AB_430834, 1:6000), HRP-conjugated anti-goat (Abcam; catalog \#Ab6741, RRID:AB_955424, 1:3000), IR dye-conjugated donkey anti-mouse 800CW (LI-COR Biosciences; catalog \#926-32213 RRID: AB_621847, 1:10,000), and IR dye-conjugated donkey anti-goat 680LT (LI-COR Biosciences, catalog \#926-68024 RRID:AB_10706168, 1:10,000).

DNA constructs. A construct encoding GFP-Shank3 was a gift from Dr. Craig Garner (Stanford University). The construct expressing shRNA (pLL3.7) was a gift from Dr. Luk Van Parijs (Massachusetts Institute of Technology). This plasmid was modified to replace the CMV promoter with a $0.4 \mathrm{~kb}$ fragment of the mouse CaMKII promoter (designated as pLLCK) that is primarily active only in excitatory neurons (Dittgen et al., 2004). Control shRNA (5'-TCGCTTGGGCGAGAGTAAG-3') was designed following Boudkkazi et al. (2014). Shank3 shRNA (5'-GGAAGT CACCAGAGGACAAGA-3') was designed following Verpelli et al. (2011). Sequences encoding shRNA were inserted into pLLCK at HpaI and XhoI restriction sites. For $\mathrm{Ca}^{2+}$ imaging studies using fura-2, the sequence encoding GFP in pLLCK was replaced with the sequence encoding the red fluorophore mApple. The Shank3 shRNA-resistant construct $\left(\right.$ Shank $3^{\mathrm{R}}$ ) was designed by introducing 6 "silent" nucleotide mutations in the target site that do not alter amino acid sequence at $\operatorname{Arg}^{1187}$, Lys $^{1188}$, Ser $^{1189}$, and Pro ${ }^{1190}$. The mAp-Shank3 construct was generated by inserting Shank3 cDNA into pmApple-C1 construct at BgIII and EcoRI restriction sites. The Shank3 shRNA-resistant construct containing a deletion of the PDZ domain (mAp-Shank $3^{\mathrm{R}-} \Delta \mathrm{PDZ}$ ) was generated by in-frame PCR deletion of the entire $270 \mathrm{bp}$ region encoding ${ }^{572}$ Iso-Val ${ }^{661}$ from mAp-Shank ${ }^{\mathrm{R}}$.

Rat $\mathrm{Ca}_{\mathrm{V}} 1.3$ complete coding sequence (GenBank accession number AF370010) was a gift from Dr. Diane Lipscombe (Brown University). A plasmid encoding $\mathrm{Ca}_{\mathrm{V}} 1.3-\mathrm{CTD}$ with an N-terminal HA-tag (HA-Ca $1.3-$ $\mathrm{CTD}$, for coimmunoprecipitation) was made by inserting rat $\mathrm{Ca}_{\mathrm{V}} 1.3 \mathrm{cDNA}$ 
encoding ${ }^{1469}$ Met-Leu ${ }^{2164}$ into the pCGNh vector, a gift from Dr. Winship Herr (Université de Lausanne). All constructs were confirmed by DNA sequencing.

Mouse forebrain homogenization and subcellular fractionation. Forebrains were dissected and fractionated as previously described (Baucum et al., 2015; Stephenson et al., 2017). Briefly, P28-P30 mice were anesthetized with isoflurane, decapitated, and forebrains were quickly dissected, cut in half down the midline, and a half forebrain was immediately homogenized in an isotonic buffer $(150 \mathrm{~mm} \mathrm{KCl}, 50 \mathrm{~mm}$ Tris $\mathrm{HCl}$, pH 7.5, 1 mм DTT, $0.2 \mathrm{~mm}$ PMSF, $1 \mathrm{~mm}$ benzamidine, $1 \mu \mathrm{M}$ pepstatin, $10 \mathrm{mg} / \mathrm{L}$ leupeptin, $1 \mu \mathrm{M}$ microcystin). The homogenate $(\sim 5 \mathrm{ml})$ was rotated end-over-end at $4^{\circ} \mathrm{C}$ for $30 \mathrm{~min}$, at which point an aliquot of the "whole forebrain" input was collected. Samples were then centrifuged at 100,000 $\times$ $g$ for $1 \mathrm{~h}$. After removing the supernatant (cytosolic S1 fraction), the pellet was resuspended in the isotonic buffer containing $1 \%(\mathrm{v} / \mathrm{v})$ Triton $\mathrm{X}-100$, triturated until homogeneous, and then rotated end-over-end at $4^{\circ} \mathrm{C}$ for $30 \mathrm{~min}$. Homogenates were then centrifuged at $10,000 \times g$ for 10 min, and the supernatant (Triton-soluble membrane S2 fraction) was removed. The second pellet (Triton-insoluble synaptic $\mathrm{P} 2$ fraction) was resuspended in isotonic buffer containing $1 \%$ Triton X-100 and 1\% deoxycholate and then sonicated. The $\mathrm{P} 2$ fraction was then mixed with $4 \times$ SDS-PAGE sample buffer or used for immunoprecipitation studies (see below).

Recombinant mouse CaMKII $\alpha$ and GST-tagged protein purification. Expression and purification of recombinant mouse CaMKII $\alpha$ have been described previously (McNeill and Colbran, 1995). GST-Shank3 constructs were created by PCR amplification of the relevant cDNA fragments for insertion between EcoR1 and BamH1 restriction sites in pGEX6P-1. GSTGluN2B was described previously (Strack et al., 2000). The vectors encoding GST fusion proteins were transformed into BL21 (DE3) pLysS bacteria cells, and proteins were purified as previously described (Robison et al., 2005a).

CaMKII autophosphorylation and GST cosedimentation assays. CaMKII $\alpha$ (1.25 $\mu \mathrm{M}$ subunit) was incubated on ice for $90 \mathrm{~s}$ with $50 \mathrm{~mm}$ HEPES, $\mathrm{pH}$ 7.5, $10 \mathrm{~mm}$ magnesium acetate, $0.5 \mathrm{~mm} \mathrm{CaCl}_{2}, 1 \mu \mathrm{M} \mathrm{CaM}$, and $1 \mathrm{~mm}$ DTT, with or without $400 \mu \mathrm{M}$ ATP (T286 autophosphorylated or basal, respectively), and reactions were terminated with $45 \mathrm{~mm}$ EDTA. Separate reactions incubated CaMKII $\alpha$ (1.25 $\mu$ M subunit) with 50 mM HEPES, pH 7.5, $10 \mathrm{~mm}$ magnesium acetate, $0.5 \mathrm{mM} \mathrm{CaCl}_{2}, 1 \mu \mathrm{M} \mathrm{CaM}, 1 \mathrm{~mm}$ DTT, with no EDTA or ATP added (Ca/CaM). The reaction was then diluted 10 -fold using $1 \times$ GST pulldown buffer $(50 \mathrm{~mm}$ Tris- $\mathrm{HCl}$, pH 7.5, $200 \mathrm{~mm} \mathrm{NaCl}$; $1 \%(\mathrm{v} / \mathrm{v})$ Triton X-100), supplemented with $10 \mathrm{~mm}$ magnesium acetate and $0.5 \mathrm{~mm} \mathrm{CaCl}_{2}$ for $\mathrm{Ca} / \mathrm{CaM}$ incubations. CaMKII $\alpha$ (125 nM subunit) was incubated with GST or GST-fusion protein (125 nM) and Pierce Glutathione Agarose beads (Thermo Fisher Scientific, catalog \#16101, 10 $\mu \mathrm{l}$ packed resin). Reactions were rocked for $1 \mathrm{~h}$ at $4^{\circ} \mathrm{C}$. Beads were washed three times with GST buffer, supplemented as described above where appropriate. Proteins were eluted with $20 \mathrm{~mm}$ glutathione, $\mathrm{pH}$ 8.0, for $10 \mathrm{~min}$ (Sigma Millipore).

Western blot analysis. Samples were resolved on 10\% SDS-PAGE gels and transferred to nitrocellulose membrane (Protran). The membrane was blocked in blotting buffer containing $5 \%$ nonfat dry milk, $0.1 \%$ Tween 20, in TBS (20 mM Tris, $136 \mathrm{~mm} \mathrm{NaCl}$ ) at pH 7.4 for 30 min at room temperature. The membrane was incubated with primary antibody (see dilutions above) in blotting buffer for $1 \mathrm{~h}$ at room temperature or overnight at $4^{\circ} \mathrm{C}$. After washing, membranes were incubated with HRPconjugated secondary antibody for $1 \mathrm{~h}$ at room temperature, washed again, and then visualized using enzyme-linked chemiluminescence using the Western Lightening Plus-ECL, enhanced chemiluminescent substrate (PerkinElmer) and visualized using Premium x-ray Film (Phenix Research Products) exposed to be in the linear response range. Images were quantified using ImageJ software (RRID:SCR_003070). Signals detected in the negative control lanes were used as background value and were subtracted from experimental lanes. Secondary antibodies conjugated to infrared dyes (LI-COR Biosciences) were used for development with an Odyssey system (LI-COR Biosciences).

Cell culture, transfection, and lysis. HEK293T cells (ATCC; catalog \#CRL-3216, RRID:CVCL_0063) were cultured and maintained in DMEM containing 10\% FBS, L-glutamine, and 1\% penicillin/streptomy- cin at $37^{\circ} \mathrm{C}$ in $5 \% \mathrm{CO}_{2}$. Cells plated on $10 \mathrm{~cm}$ dishes were transfected with 5-10 $\mu \mathrm{g}$ of DNA. After $24-48 \mathrm{~h}$, cells were rinsed in ice-cold PBS and lysed in ice-cold lysis buffer ( $150 \mathrm{~mm} \mathrm{NaCl}, 25 \mathrm{~mm}$ Tris-HCl, pH 7.5, $1 \%$ Triton X-100, 2 mм EDTA, 2 mм EGTA, 1 mм DTT, 0.2 mм PMSF, 1 mм benzamidine, $10 \mu \mathrm{g} / \mathrm{ml}$ leupeptin, and $10 \mu \mathrm{M}$ pepstatin).

Immunoprecipitation. Mouse forebrain fractions or HEK293T cell lysates were incubated with mouse anti-CaMKII $\alpha$ (Thermo Fisher Scientific, catalog \#MA1-048), rabbit anti-Shank3 (Bethyl, catalog \#A304178A RRID:AB_2621427), or rabbit anti-GFP (Thermo Fisher Scientific, catalog \#A-11222 RRID:AB_221569) and rocked end-over-end at $4^{\circ} \mathrm{C}$ for $1 \mathrm{~h}$ with $10 \mu \mathrm{l}$ prewashed Dynabeads Protein G (Thermo Fisher Scientific, catalog \#10002D, for mouse) or Protein A (Thermo Fisher Scientific, catalog \#10001D, for rabbit). HEK293T lysates were supplemented with $1.5 \mathrm{~mm} \mathrm{CaCl}_{2}$ and $1 \mu \mathrm{M}$ calmodulin (final concentrations) to partially activate CaMKII during immunoprecipitation. The beads were isolated magnetically and washed three times using lysis buffer before eluting proteins using $2 \times$ SDS-PAGE sample buffer. Inputs and immune complexes were immunoblotted side by side as indicated (see Western blot analysis).

Colocalization studies. STHdh ${ }^{\mathrm{Q} 7 / \mathrm{Q} 7}$ cells (RRID:CVCL_M590) (Trettel et al., 2000), a gift from Dr. Aaron Bowman (Vanderbilt University), were cultured and maintained in DMEM containing 10\% FBS, L-glutamine, $1 \%$ penicillin/streptomycin, and $400 \mu \mathrm{g} / \mathrm{ml} \mathrm{G} 418$ (Mediatech) at $33^{\circ} \mathrm{C}$ in $5 \% \mathrm{CO}_{2}$. Cells were plated onto $15 \mathrm{~mm}$ coverslips pretreated with polyD-lysine in 12-well plates and transfected with $3 \mu \mathrm{g}$ of DNA overnight. Media was then removed and cells were incubated in serum-free DMEM containing either $0.49 \%$ DMSO (Pierce) or a differentiation medium (serum-free DMEM supplemented with $10 \mathrm{ng} / \mathrm{ml}$ fibroblast growth factor) (Promega), $240 \mu \mathrm{M}$ isobutylmethylxanthine (Sigma Millipore), 20 $\mu \mathrm{M}$ 12-O-tetradecanoylphorbol-13-acetate (Sigma Millipore), $48.6 \mu \mathrm{M}$ forskolin (Sigma Millipore), and $5 \mu \mathrm{M} \mathrm{DA}$ (Sigma Millipore). After 8-14 $\mathrm{h}$, cells were fixed in ice-cold $4 \% \mathrm{PFA}-4 \%$ sucrose in $0.1 \mathrm{M} \mathrm{PB}, \mathrm{pH} 7.4$, for $3 \mathrm{~min}$ and $-20^{\circ} \mathrm{C}$ methanol for $10 \mathrm{~min}$. Coverslips were mounted on microscope slides using ProLong Gold antifade reagent with DAPI (Thermo Fisher Scientific, catalog \#P36931). Images were collected using a Carl Zeiss 880 inverted confocal microscope using $63 \times$ objective. Thresholding and intensity correlation analysis to compare normalized pixel intensities in each color channel were performed using ImageJ as previously described (Baucum et al., 2010). GFP and mApple channels were automatically thresholded before calculating the intensity correlation quotient (ICQ). This method interprets ICQ values in the following ranges: $0<\mathrm{ICQ} \leq 0.5$, dependent overlap of fluorescent signals; ICQ $=$ 0 , random overlap of fluorescent signals; and $0>$ ICQ $\geq-0.5$, segregation of fluorescent signals (Li et al., 2004).

Primary hippocampal neuronal cultures and neuronal stimulation assay. Dissociated hippocampal neurons were prepared from E18 Sprague Dawley rat embryos, as previously described (Shanks et al., 2010). Sex of the embryos was not determined. Neurons were transfected at 7-9 DIV using Lipofectamine 2000 following the manufacturer's directions (Thermo Fisher Scientific). A total of $1 \mu \mathrm{g}$ of DNA was transfected for each well of a 12-well plate for $2-3 \mathrm{~h}$ before switching back to conditioned media. At DIV 13-14, neurons were preincubated for $2 \mathrm{~h}$ with $5 \mathrm{~K} \mathrm{Ty}$ rode's solution (150 mM NaCl, $5 \mathrm{~mm} \mathrm{KCl,} 2 \mathrm{mM} \mathrm{CaCl}_{2}, 2 \mathrm{~mm} \mathrm{MgCl}$, 10 mm glucose, and $10 \mathrm{~mm}$ HEPES, pH 7.5, $\sim 313 \mathrm{mOsm}$ ) with $1 \mu \mathrm{M}$ TTX, $10 \mu \mathrm{M}$ APV, and $50 \mu \mathrm{M}$ CNQX to suppress intrinsic neuronal activity by blocking sodium channels, NMDARs and AMPARs, respectively. Neurons were then treated with either $5 \mathrm{~K}$ Tyrode's or $40 \mathrm{~K}$ Tyrode's solution (adjusted to $40 \mathrm{~mm} \mathrm{KCl}$ and $115 \mathrm{~mm} \mathrm{NaCl}$, with all three inhibitors present) for $90 \mathrm{~s}$. For the analysis of pCREB levels, neurons were immediately fixed using ice-cold $4 \%$ PFA- $4 \%$ sucrose in $0.1 \mathrm{M} \mathrm{PB}, \mathrm{pH} 7.4$, for $3 \mathrm{~min}$ and $-20^{\circ} \mathrm{C}$ methanol for $10 \mathrm{~min}$. For the analysis of c-Fos expression, Tyrode's solution was aspirated after $90 \mathrm{~s}$ and replaced with conditioned media for $3 \mathrm{~h}$ before fixation. Fixed neurons were washed three times with PBS, permeabilized with PBS $+0.2 \%$ Triton X-100, and then incubated with blocking solution for $1 \mathrm{~h}$ : $1 \times$ PBS, $0.1 \%$ Triton X-100 $(\mathrm{v} / \mathrm{v}), 2.5 \%$ BSA (w/v), 5\% normal donkey serum (w/v), 1\% glycerol $(\mathrm{v} / \mathrm{v})$. Neurons were then incubated with blocking solution overnight with primary antibodies: rabbit anti-pCREB (Cell Signaling Technology, catalog \#9198, RRID:AB_2798432, 1:1000) or rabbit anti-c-Fos (EMD 
Millipore, catalog \#ABE457, RRID:AB_310107, 1:500), and mouse antiCaMKII $\alpha$ 6G9 (Thermo Fisher Scientific, catalog \#MA1-048, 1:1000). The following day, neurons were washed three times in PBS $+0.2 \%$ Triton X-100 and then incubated with blocking solution for $1 \mathrm{~h}$ with secondary antibodies: donkey anti-rabbit 647 AlexaFluor-647 (Thermo Fisher Scientific, catalog \#A-31573, RRID:AB_10891079) and donkey anti-mouse AlexaFluor-546 (catalog \#A-10036, RRID:AB_2534012). Neurons were washed with PBS three times and mounted on slides using Prolong Gold Antifade Mountant with DAPI.

Neuronal imaging and quantification. The experimenter was blinded to the transfection conditions by coding the culture dishes before microscopy and image analysis. Images were collected using a Carl Zeiss 880 inverted confocal microscope with a $40 \times / 1.30$ Plan-Neofluar oil lens. The binocular lens was used to identify transfected neurons based on EGFP expression from the shRNA construct. In experiments with mApShank3 rescue, mAp expression was also confirmed in EGFP-positive cells. The DAPI channel was then used to focus on the $z$-plane that yielded the highest DAPI signal (one that presumably runs through the nuclei) for image acquisition. Images were then collected in all channels, and MetaMorph Microscope Automation and Image Analysis Software (Molecular Devices, RRID:SCR_002368) was used to quantify pCREB or c-Fos signals. Briefly, nuclei were identified by thresholding the DAPI channel to create and select the nuclear ROIs. The ROIs were then transferred to other channels to measure the average pCREB or c-fos intensity. ROIs were collected from transfected (EGFP-positive) neurons and nearby nontransfected (EGFP-negative) neurons. The relative intensity was calculated as $\left[\left(\right.\right.$ channel $^{\mathrm{x}}-$ channel $\left.^{5 \mathrm{~K}}\right) /\left(\right.$ channel $^{40 \mathrm{~K}}-$ channel $\left.\left.^{5 \mathrm{~K}}\right)\right]$, where channel ${ }^{\mathrm{x}}$ is the signal being calculated, and channel ${ }^{5 \mathrm{~K}}$ and channel ${ }^{40 \mathrm{~K}}$ are the average signals of the $5 \mathrm{~K}$ and $40 \mathrm{~K}$ conditions in that batch of cultured neurons, respectively. Sample size was calculated based on previous experimental design (Wang et al., 2017). Data shown were collected from images of the indicated total number of neurons from 3 to 5 independent cultures.

Neuronal $\mathrm{Ca}^{2+}$ imaging. Dissociated rat hippocampal neurons were cultured in $35 \mathrm{~mm}$ glass-bottom dishes (Cellvis, catalog \#D35-10-1.5-N) coated with $2.5 \mu \mathrm{g} / \mathrm{ml} \mathrm{laminin} \mathrm{(Roche} \mathrm{Diagnostics)} \mathrm{and} 37.5 \mu \mathrm{g} / \mathrm{ml}$ polyL-lysine (Sigma Millipore), as described previously (Sala et al., 2003). Cultures were transfected with a total of $2.5 \mu \mathrm{g}$ of DNA/dish after DIV 8, as described above. To allow for $\mathrm{Ca}^{2+}$ imaging using fura-2, shRNA constructs were reengineered to coexpress an mApple fluorescent reporter rather than GFP. Transfected neurons were imaged on DIV 12-14. Cells were incubated for $30 \mathrm{~min}$ at $37^{\circ} \mathrm{C}$ in $5 \% \mathrm{CO}_{2}$ in conditioned culture medium (see above) supplemented with $2 \mu \mathrm{M}$ fura- 2 acetoxymethyl ester AM (Thermo Fisher Scientific, catalog \#F1221) and then incubated in 5K Tyrode's solution with TTX, APV, and CNQX (see above) for $5 \mathrm{~min}$ at $37^{\circ} \mathrm{C}$. Cells were incubated in $2.5 \mathrm{ml}$ solution and perfused at a flow rate of $2 \mathrm{ml} / \mathrm{min}$ at $32^{\circ} \mathrm{C}$ with identical buffer for at least $300 \mathrm{~s}$; then flow was changed to $40 \mathrm{~K}$ Tyrode's solution with TTX, APV, and CNQX for at least $150 \mathrm{~s}$. Intracellular $\mathrm{Ca}^{2+}$ was measured every $5 \mathrm{~s}$ as a ratio of fura-2 emission $(510 \mathrm{~nm})$ by excitation at 340 and $380 \mathrm{~nm}\left(\mathrm{~F}_{340} / \mathrm{F}_{380}\right)$ using an Eclipse Ti2 microscope (Nikon) equipped with an epifluorescence illuminator (Sutter Instrument), a Prime 95B equipped with 25 $\mathrm{mm}$ CMOS sensors camera (Photometrics Scientific), and Elements software (Nikon; RRID:SCR_014329). Transfected (mApple-positive) cell somas were selected as ROIs using Elements software (Nikon), and $\mathrm{Ca}^{2+}$ responses were quantified as a function of time by the change in fura-2 fluorescence ratio above baseline $\left(\Delta \mathrm{F}=\left(\mathrm{F}_{340} / \mathrm{F}_{380}\right) /\left(\mathrm{F}_{340} / \mathrm{F}_{380}\right)_{\text {baseline }}\right)$. Daily averages from 12 to 90 transfected control and Shank3 shRNA cells were generated from four independent biological replicates. Areas under the curve of the averaged fura- 2 responses during the first $90 \mathrm{~s}$ of stimulation were quantified as an indicator of the change in intracellular $\mathrm{Ca}^{2+}$ for each replicate.

Statistics. Statistical tests and parameters are indicated in figure legends. Differences were considered significant if $p \leq 0.05$. Sample sizes for each experiment are based on previously published studies from our laboratory and standards in the field. The experimenter was blind to the transfection condition when analyzing the levels of pCREB or c-Fos for Figures 6-8. Data are mean \pm SEM with individual data points indicated.

\section{Results}

\section{CaMKII $\alpha$ and Shank 3 interact in the mouse forebrain}

Our previous proteomics study detected numerous peptides originating from the Shank3 protein in immunoprecipitated CaMKII complexes isolated from solubilized synaptic fractions of mouse forebrain (Baucum et al., 2015). To extend this observation, we first compared the distribution of Shank3 and CaMKII across cytosolic (S1), Triton-soluble membrane (S2), and Tritoninsoluble synaptic (P2) fractions isolated from mouse forebrain extracts. The Shank3 antibody detected two major bands in whole mouse forebrain extracts: the expected $\sim 180 \mathrm{kDa}$ band, plus an $\sim 125 \mathrm{kDa}$ band. Both Shank3 bands were undetectable in $\mathrm{S} 1$ or $\mathrm{S} 2$ fractions and were relatively enriched in the $\mathrm{P} 2$ fraction, similar to other synaptic proteins, such as PSD-95 (Fig. 1A, left). Since Shank3 undergoes complex transcriptional and posttranscriptional regulation through intragenic promoters and alternative splicing (Wang et al., 2011; Waga et al., 2014), the two bands may represent different Shank3 variants. In contrast, similar levels of CaMKII were detected in the S2 and P2 fractions, with lower levels in S1, consistent with our prior studies (Gustin et al., 2011). Thus, a subpopulation of CaMKII and essentially all of the Shank3 are present in mouse forebrain subcellular fractions enriched in synaptic proteins in WT mice.

To further investigate the association of Shank3 with CaMKII $\alpha$, synaptic P2 fractions were isolated in parallel from WT or CaMKII $\alpha$-KO littermates (as a negative control), solubilized by sonication in sodium deoxycholate plus Triton X-100 to at least partially disrupt the postsynaptic density (see Materials and Methods), and incubated with a control IgG antibody or a CaMKII $\alpha$-specific monoclonal antibody. Immunoblotting confirmed that CaMKII $\alpha$-KO mice do not express CaMKII $\alpha$, and revealed that the levels of Shank3 in whole forebrain lysates (both major bands) and the distribution of Shank3 between the S1, S2, and $\mathrm{P} 2$ fractions was similar in WT and CaMKII $\alpha$-KO mice (Fig. $1 A, B$, left). Shank3 was readily detected in CaMKII $\alpha$ complexes isolated from WT mouse forebrain, but not from CaMKII $\alpha$-KO mouse forebrain, and not in any samples isolated using a control IgG (Fig. 1C). Interestingly, the ratio of the higher and lower molecular weight Shank3 bands was consistently higher in CaMKII immune complexes than in the $\mathrm{P} 2$ input, perhaps suggesting that CaMKII preferentially interacts with the larger Shank3 protein. Together, these data demonstrate that Shank3 is specifically associated with CaMKII $\alpha$ complexes in mouse brain extracts.

We next tested for reciprocal coimmunoprecipitation of CaMKII $\alpha$ with complexes isolated using a Shank3 antibody. Since interactions between CaMKII and other proteins are often enhanced by Thr286 autophosphorylation, we compared the association of CaMKII $\alpha$ with Shank3 in solubilized synaptic P2 fractions isolated from WT mice and CaMKII $\alpha^{\text {T286A }}$ mice. The knock-in mutation of Thr286 to Ala in CaMKII $\alpha^{\text {T286A }}$ mice prevents CaMKII regulation by Thr286 autophosphorylation (Giese et al., 1998). Immunoblotting confirmed that whole forebrain lysates from WT and CaMKII $\alpha^{\text {T286A }}$ mice contain similar total levels of CaMKII $\alpha$ and Shank3 (both major bands) (Fig. 1B, right). Moreover, the distribution of Shank3 between S1, S2, and $\mathrm{P} 2$ fractions was similar in WT and CaMKII $\alpha^{\mathrm{T} 286 \mathrm{~A}}$ mice (Fig. $1 A$ ), although CaMKII $\alpha$ was partially redistributed from P2 to S2 fractions in CaMKII $\alpha^{\text {T286A }}$ mice (Fig. $1 A$ ), as previously reported (Gustin et al., 2011). Shank3 immune complexes isolated from WT and CaMKII $\alpha^{\text {T286A }}$ mice contained similar levels of Shank3, but there was a substantial reduction in levels of coimmunoprecipitated CaMKII $\alpha$ from CaMKII $\alpha^{\text {T286A }}$ compared with WT tis- 
A

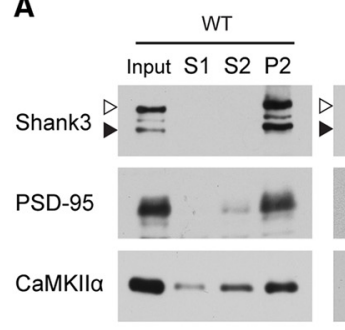

C IP from WT or CaMKIla-KO

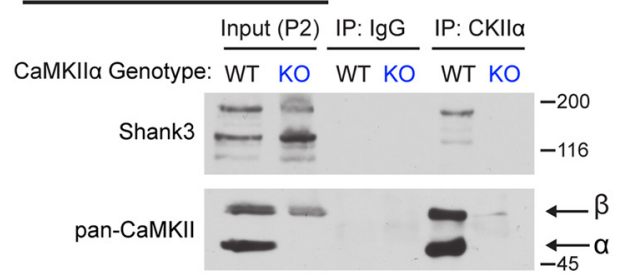

B Total Shank3 Protein Levels

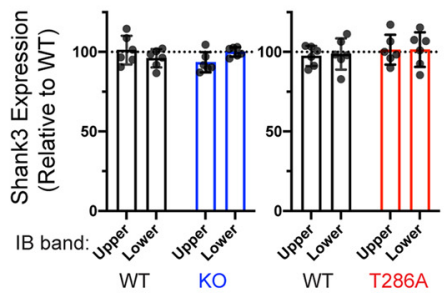

D IP from WT or CaMKIII ${ }^{\top 286 A}$

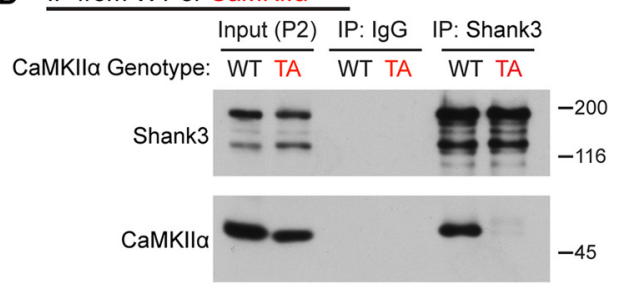

Figure 1. Reciprocal coimmunoprecipitation of Shank3 and CaMKIll $\alpha$ from mouse forebrain extracts. A, Whole forebrain lysates (Input), cytosolic (S1), membrane-associated (S2), and synaptic (P2) subcellular fractions from WT, CaMKIl $\alpha$-K0, and CaMKII $\alpha{ }^{\text {T286A }}$ mice were immunoblotted for localization of Shank3 (Cell Signaling Technology antibody), PSD-95, and CaMKIl $\alpha$. The Shank3 antibody detected two bands (open and solid arrowheads) that are primarily localized in P2 fractions, which were also enriched for CaMKII $\alpha$. B, The upper and lower Shank3 bands in whole forebrain lysates from WT and CaMKII $\alpha$-KO mice (left) or WT and CaMKII $\alpha^{\text {T286A }}$ mice (right) were quantified. Signals were corrected for protein loading based on Ponceau-stained membranes and normalized to the levels of the upper Shank3 band in WT samples. C, Synaptic (P2) fractions from WT or CaMKII $\alpha$-KO mouse forebrains were immunoprecipitated using control lgG or CaMKII $\alpha$-specific antibodies, and immunoblotted using Shank3 (Cell Signaling Technology antibody) and pan-CaMKII antibodies. Arrows indicate CaMKII $\alpha$ and CaMKII $\beta$ bands. CaMKII $\alpha$ and coimmunoprecipitated Shank3 were not detected in samples isolated from CaMKII $\alpha$-K0 mice. D, Synaptic (P2) fractions from WT or CaMKII $\alpha{ }^{\text {T286A }}$ mouse forebrains were immunoprecipitated using control lgG or Shank3 (Bethyl) antibodies, and immunoblotted using Shank3 (Cell Signaling Technology) and CaMKIIl $\alpha$ antibodies. The levels of coprecipitated CaMKIll $\alpha$ from CaMKIll $\alpha^{\text {T286A }}$ mice were significantly reduced ( $93 \pm 4 \%$ reduction in lane 6 compared with lane $5, n=3 . p<0.001$, one-sample Student's $t$ test with equal variance compared with theoretical value of 100). All immunoblots are representative of $\geq 3$ biological replicates.

sue (Fig. $1 D$, compare CaMKII $\alpha$ signal in lanes 5 and 6$)(93 \pm 4 \%$ reduced compared with WT, $p=0.0008$, one-sample Student's $t$ test with equal variance compared with the theoretical normalized WT value of $100, n=3)$. In combination, these data indicate that Shank3 interacts directly or indirectly with CaMKII in deoxycholate-solubilized synaptic fractions of mouse brain, and that this association is regulated by Thr286 autophosphorylation of CaMKII.

\section{T286-autophosphorylated CaMKII $\alpha$} directly binds to Shank3 (829-1130) To determine whether CaMKII $\alpha$ directly interacts with Shank3, we first expressed and purified a series of six nonoverlapping GST fusion proteins spanning the full length of Shank3 (Figs. 2A,B). Some of the purified proteins contained proteolytic degradation fragments, and the fulllength proteins are denoted by asterisks in Figure $2 B$. Since coimmunoprecipitation of CaMKII $\alpha$ with Shank3 from brain extracts was strongly reduced in the absence of Thr286 autophosphorylation, each purified GST fusion protein was incubated with purified Thr286-autophosphorylated CaMKII $\alpha$, and complexes were isolated using glutathione agarose. A GST fusion protein containing the CaMKII binding domain of the NMDAR GluN2B subunit (residues 1260-1309), a well-
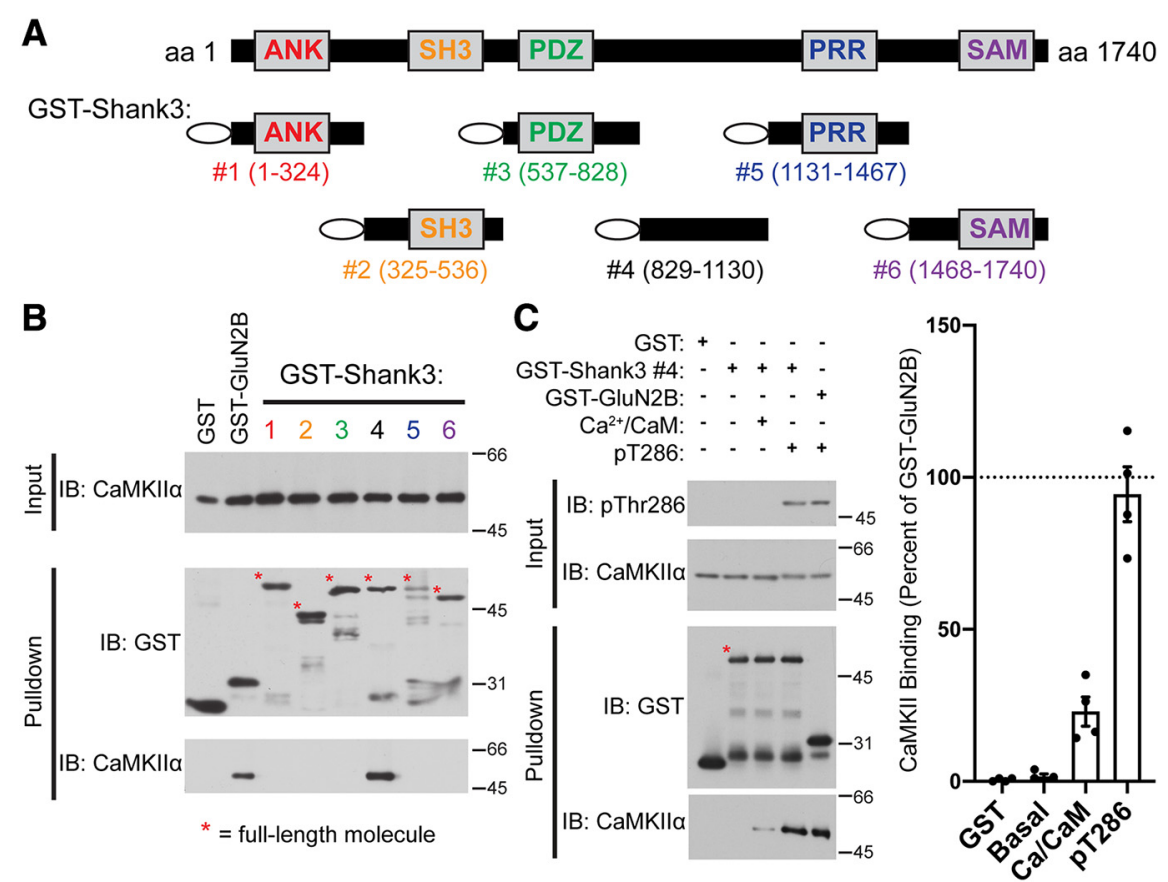

Figure 2. T286-phosphorylated CaMKIl $\alpha$ specifically binds to Shank3 (829-1130). $A$, Domain structure of full-length Shank3 and the six GST-Shank3 fusion proteins used in these studies that span the entire Shank3 protein. Gray boxes represent canonical Shank3 domains. Residue numbers are listed in parentheses. ANK, Ankyrin repeats domain; SH3, Src homology 3 domain; PDZ, PSD95/Dlg1/zo-1 domain; PRR, proline-rich region containing binding sites for Homer and Cortactin; SAM, sterile $\alpha$ motif involved in multimerization of Shank3. B, Glutathione agarose cosedimentation assay shows that preactivated (Thr286autophosphorylated) CaMKII $\alpha$ specifically binds to GST-Shank3 \#4 (829-1130) and positive control GST-GluN2B (1260-1309). *Full-length GST fusion proteins on the GST immunoblot. C, Glutathione agarose cosedimentation assay shows that, in the absence of $\mathrm{Ca}^{2+} / \mathrm{CaM}$ binding or Thr286 autophosphorylation, CaMKIl $\alpha$ (Basal) does not bind to GST-Shank3 \#4; in vitro binding of CaMKIll $\alpha$ is partially supported by $\mathrm{Ca}^{2+} / \mathrm{CaM}$ binding (Ca/CaM), and maximally enhanced by pT286 autophosphorylation. Bar graph represents levels of each form of CaMKII bound to GST-Shank3 \#4 (or the GST negative control) (mean \pm SEM) relative to levels of pT286-CaMKII $\alpha$ binding to GST-GluN2B. Immunoblots are representative of three or four biological replicates. 
established CaMKAP (Strack and Colbran, 1998), was used as a positive control. Similar amounts of activated CaMKII $\alpha$ bound to a GST-Shank3 fusion protein containing residues 829-1130 (Fig. 2, GST-Shank3 \#4) and to GST-GluN2B, but there was no consistently detectable interaction with any other Shank3 fragment (Fig. 2B).

CaMKII is initially activated by $\mathrm{Ca}^{2+} / \mathrm{CaM}$ binding alone followed by Thr286 autophosphorylation, which is sufficient to maintain the activated conformation, even following dissociation of $\mathrm{Ca}^{2+} / \mathrm{CaM}$. Since CaMKAPs display distinct preferences for binding to various active and inactive conformations of CaMKII, we tested the binding of different CaMKII $\alpha$ conformations to GST-Shank3 (829-1130) in parallel (Fig. 2C). There was no detectable binding of inactive CaMKII to GST-Shank3 (829-1130) (Fig. 2C), suggesting that CaMKII activation is essential for binding to this fragment. Inactive CaMKII $\alpha$ also did not bind to any other GST-Shank3 proteins compared with GST negative control under these conditions (data not shown). GST-Shank3 (8291130 ) bound to the active conformations of CaMKII $\alpha$ induced by $\mathrm{Ca}^{2+} / \mathrm{CaM}$ binding alone or by Thr286 autophosphorylation, but $\mathrm{Ca}^{2+} / \mathrm{CaM}$ binding alone only partially supported binding $(23 \pm 5 \%$; mean \pm SEM). In combination, these data show that activated CaMKII $\alpha$ can directly bind to a central, poorly characterized domain in the Shank3 protein, and that Thr286 autophosphorylation significantly enhances the interaction.

\section{A Shank3 tribasic residue motif is required for CaMKII binding}

Residues $829-1130$ of Shank3 connect the canonical PDZ domain to the proline-rich motif, but the functional role(s) of this region is poorly understood (Naisbitt et al., 1999; Tu et al., 1999). To identify CaMKII binding determinants within this domain, we initially generated and purified three smaller GST-Shank 3 fusion proteins (Fig. 3A). Similar amounts of Thr286-autophosphorylated CaMKII bound to GST-Shank3 (931-1014) (protein 4b in Fig. $3 A$ ) and GST-Shank3 (829-1130), but there was no detectable interaction with GST-Shank3 proteins containing residues $829-$ 930 (protein 4a) or residues 1015-1130 (protein 4c) (Fig. 3B). Examination of the amino acid sequence of Shank3 residues 9311014 revealed a region sharing some similarity with recently characterized CaMKII binding domains in the N-terminal domain of the LTCC $\mathrm{Ca}_{\mathrm{V}} 1.3 \alpha$ subunit (Wang et al., 2017) and the C-terminal domain (CTD) of mGlu5 (Marks et al., 2018) (Fig. $3 A$ ). Since mutation of the tribasic residue motifs in $\mathrm{Ca}_{\mathrm{V}} 1.3$ $\left({ }^{83}\right.$ Arg-Lys-Arg $\left.{ }^{85}\right)$ and mGlu5 ( ${ }^{866}$ Lys-Arg-Arg $\left.{ }^{868}\right)$ prevented CaMKII $\alpha$ binding, we mutated the conserved Shank3 ${ }^{949} \mathrm{Arg}$ Arg-Lys ${ }^{951}$ motif to Ala residues within GST-Shank3 (8291130). This RRK/AAA mutation essentially abrogates binding of Thr286-autophosphorylated CaMKII $\alpha$ (Fig. 3C). These data indicate that ${ }^{949}$ Arg-Arg-Lys ${ }^{951}$ in Shank3 is essential for the direct binding of Thr286-autophosphorylated CaMKII in vitro.

\section{The RRK/AAA mutation of full-length Shank3 disrupts binding to CaMKII but not to $\mathrm{Ca}_{\mathrm{V}} 1.3$}

To determine whether the ${ }^{949} \mathrm{Arg}$-Arg-Lys ${ }^{951}$ motif is essential for association of CaMKII with full-length Shank3, we generated the RRK/AAA mutant in GFP-tagged full-length Shank3 (GFPShank3-AAA). CaMKII $\alpha$ was coexpressed with GFP, GFPShank3-WT, or GFP-Shank3-AAA in HEK293T cells. Excess $\mathrm{Ca}^{2+} / \mathrm{CaM}$ was added to the cell lysates to activate $\mathrm{CaMKII} \alpha$, and a GFP antibody was used for immunoprecipitation. Similar robust GFP protein signals were detected in samples immunoprecipitated from each lysate; CaMKII $\alpha$ was detected in immune complexes con-
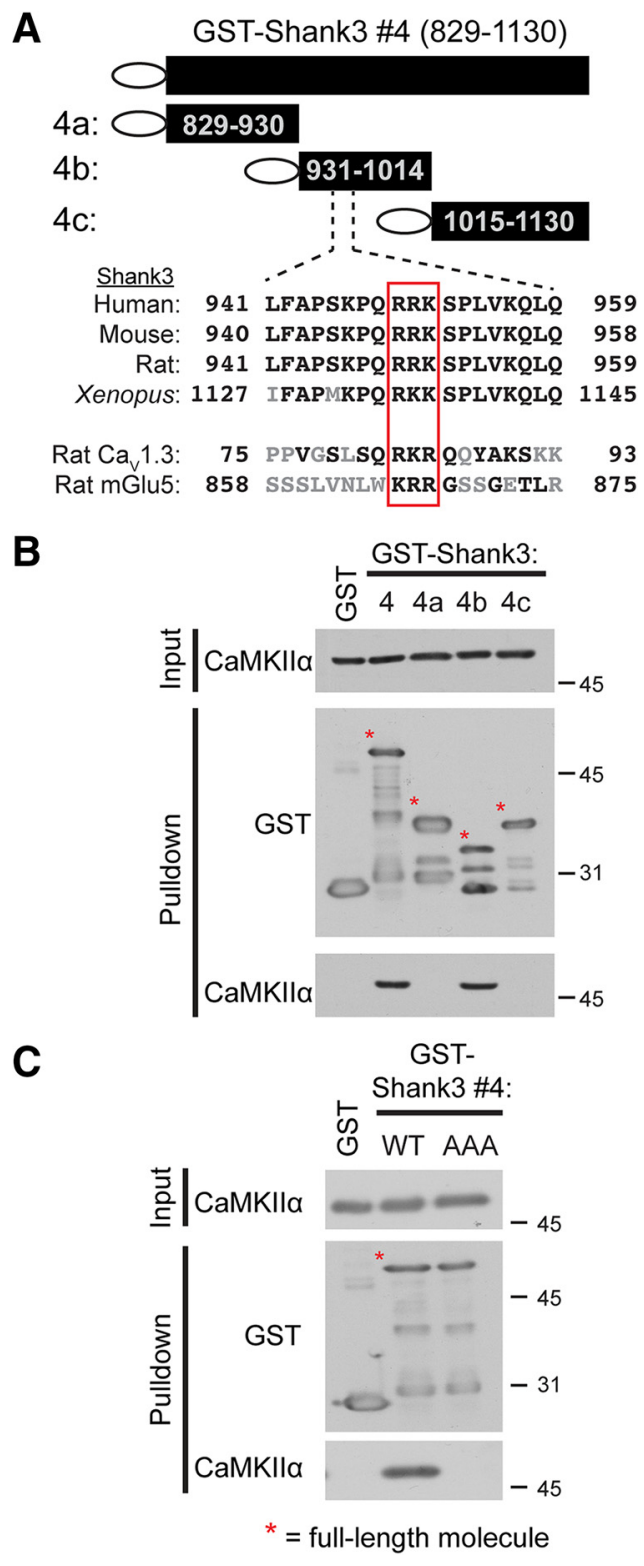

Figure 3. Characterization of the CaMKII binding motif in Shank3. A, Top, Diagram of 3 truncations used to map the CaMKII interaction site within GST-Shank3 \#4 (829-1130). Bottom, Sequence alignment of human Shank3 residues 941-959 with the corresponding Shank3 residues in other species and the CaMKII binding domain in the N-terminal domain of the Rattus norvegicus $\mathrm{Ca}_{\mathrm{v}} 1.3 \alpha 1$ subunit (Wang et al., 2017) and the C-terminal tail of the Rattus norvegicus mGlu5 (Marks et al., 2018). Black represents conserved residues. Gray represents dissimilar residues. Red box represents the conserved tribasic residue motif. $\boldsymbol{B}$, Glutathione agarose cosedimentation assay comparing binding of activated CaMKII $\alpha$ to GST-Shank3 \#4 (829 1130 ) and 3 nonoverlapping fragments (4a, 4b, and 4c). *Full-length GST fusion proteins. GST-Shank3 \#4 (829-1130) and \#4b (931-1014) bind similar amounts of pT286autophosphorylated (aMKII $\alpha$, but there is no detectable binding to the other Shank3 fragments. C, Mutation of amino acids ${ }^{949}{ }^{R R K}{ }^{951}$ to AAA in GST-Shank3 \#4 (829-1130) blocks CaMKII $\alpha$ binding in glutathione agarose cosedimentation assay ( $98 \pm 4 \%$ reduced compared with WT, $n=3, p<0.001$, one-sample Student's $t$ test with equal variance compared with theoretical value of 100). All immunoblots are representative of three biological replicates.

taining GFP-Shank3-WT, but not GFP-negative control or GFPShank3-AAA complexes (Fig. $4 A$ ). Thus, the ${ }^{949}$ Arg-Arg-Lys ${ }^{951}$ motif in Shank3 is required for CaMKII $\alpha$ association with the full length protein. 
A
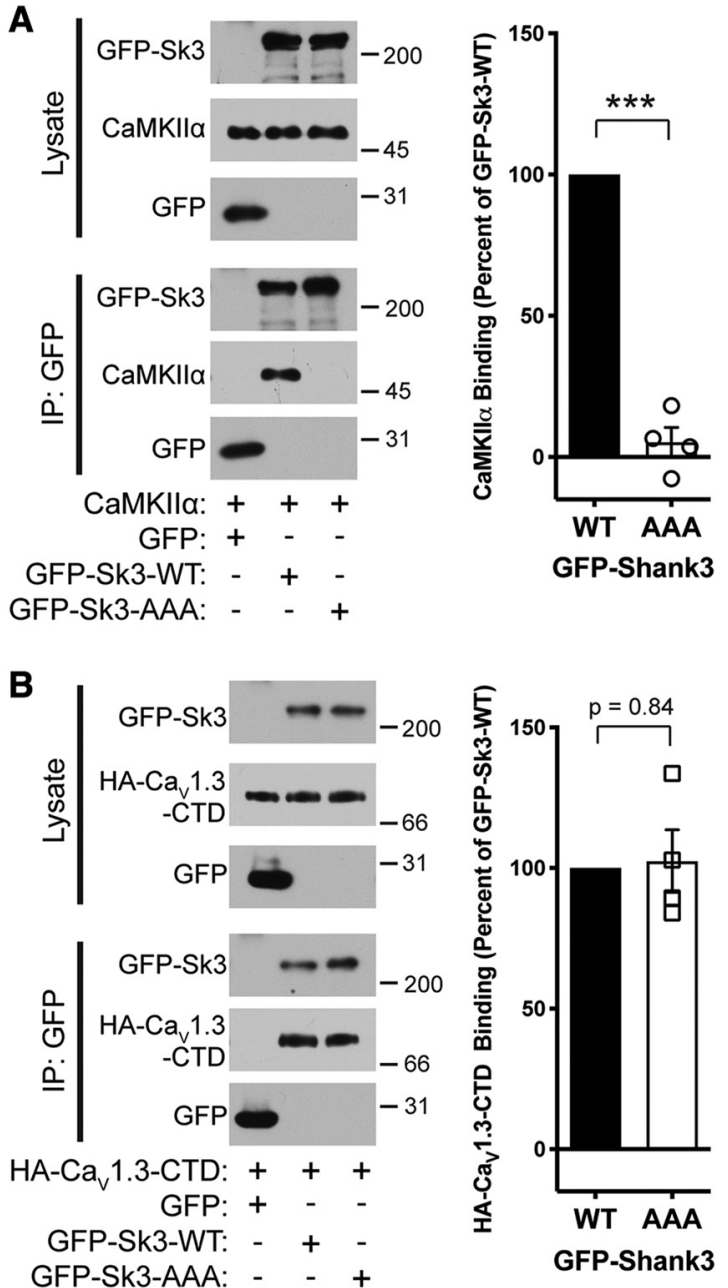

Figure 4. A Shank $3{ }^{949}{ }^{\text {RRK }}{ }^{951}$ to AAA mutation disrupts association with CaMKII $\alpha$ but does not with the $\mathrm{Ca}_{V} 1.3$ CTD. A, Soluble fractions of HEK293T cells expressing CaMKIll $\alpha$ with GFP or GFP-Shank3 (WT or ${ }^{949}$ RRK $^{951}$ to AAA mutant) were immunoprecipitated using a GFP antibody. Coprecipitation of CaMKII $\alpha$ with GFP-Shank3-AAA is significantly reduced by $95 \pm 10 \%$ compared with GFP-Shank3-WT. ${ }^{* * *} p<0.001$ (one-sample Student's $t$ test with equal variance compared with a theoretical value of 100). $\boldsymbol{B}$, Soluble fractions of HEK293T cells expressing GFP or GFP-Shank3 (WT or AAA) with the HA-tagged CTD of the $\mathrm{Ca}_{\mathrm{v}} 1.3 \alpha 1$ subunit (HA-Ca $1.3-$ CTD) were immunoprecipitated as in $\boldsymbol{A}$. The AAA mutation has no significant effect on the coprecipitation of HA-Ca 1 1.3-CTD $(p=0.84)$. All immunoblots are representative of four biological replicates. Error bars indicate the mean \pm SEM.

To test the specificity of the RRK/AAA mutation in Shank3, we performed a similar coimmunoprecipitation experiment using an HA-tagged CTD of the L-type calcium channel $\mathrm{Ca}_{\mathrm{V}} 1.3 \alpha$ subunit, which can interact with the nearby PDZ domain (residues 572-661) of Shank3 (Zhang et al., 2005). Lysates of HEK293T cells expressing HA-Ca $\mathrm{V}_{\mathrm{V}}$ 1.3-CTD and GFP, GFP-Shank3-WT, or GFP-Shank3AAA were immunoprecipitated using a GFP antibody. Similar amounts of the HA-Ca $1.3-\mathrm{CTD}$ coimmunoprecipitated with both GFP-Shank3-WT and GFP-Shank3-AAA immune complexes, but not with GFP alone (Fig. $4 B$ ). Thus, the interaction of $\mathrm{Ca}_{\mathrm{V}} 1.3$ with Shank3 is not affected by mutation of the ${ }^{949} \mathrm{Arg}$ Arg-Lys ${ }^{951}$ motif, indicating that this mutation does not have broader (nonspecific) effects on the association of other proteins with GFP-Shank3.

The RRK/AAA mutation of GFP-Shank3 disrupts colocalization with mApple-CaMKII $\alpha$

To investigate whether Shank3 interacts with CaMKII $\alpha$ in intact cells, we performed a series of colocalization experiments in
STHdh ${ }^{+/+}$cells. This striatal progenitor cell line can be pharmacologically induced to initiate a neuronal differentiation program (see Materials and Methods), extending neurite-like outgrowths containing microtubule-associate protein 2, a dendritic marker (Trettel et al., 2000). However, STHdh ${ }^{+/+}$ cells do not express detectable levels of endogenous Shank3 or CaMKII $\alpha$ before or after differentiation (Fig. $5 \mathrm{~A}$, left, middle). Therefore, they represent an excellent heterologous cell model to explore the colocalization of Shank3 and CaMKII $\alpha$ in a neuron-like environment, without potentially confounding effects of high levels of endogenous CaMKII $\alpha$, Shank3, or other CaMKAPs that might compete for the interactions of expressed proteins.

Since our in vitro studies indicate that CaMKII activation and Thr286 autophosphorylation are a critical modulator of Shank3 binding, we first investigated colocalization of GFP-Shank3-WT with mAp-CaMKII $\alpha$-WT, mAp-CaMKII $\alpha$-T286D (phosphomimetic), or mAp-CaMKII $\alpha$-T286A (phospho-null) in fixed, differentiated cells. GFP-Shank3-WT (green channel) was concentrated in puncta in the soma and in neurite-like outgrowths (Fig. $5 B$ ), but the appearance of the mApple fluorescence (magenta) varied with the CaMKII $\alpha$ Thr286 mutation. Signals from mAp-CaMKII $\alpha$-WT and CaMKII $\alpha$-T286D displayed punctate characteristics in the soma and in neurite-like outgrowths that appeared to partially overlap with GFP-Shank3-WT punctae, whereas the signal from mAp-CaMKII $\alpha$-T286A was generally diffuse throughout the cell, and the limited number of visible punctae did not align with GFP-Shank3-WT punctae (Fig. $5 B$, inset, neurite-like outgrowths). To provide an unbiased assessment of the colocalization of the mApple and GFP signals across multiple cells, we analyzed images of whole transfected cells using image correlation analysis, which quantifies colocalization on an ICQ scale from -0.5 (mutually exclusive signals) to 0.5 (perfect colocalization), with 0 representing random overlap (Li et al., 2004). The ICQ values for overlap of GFP-Shank3-WT with mApCaMKII $\alpha$-WT, mAp-CaMKII $\alpha$-T286D, and mAp-CaMKII $\alpha$ T286A were $0.29 \pm 0.02,0.36 \pm 0.02$, and $0.17 \pm 0.02$, respectively (Fig. $5 C$ ). These data confirm that mAp-CaMKII $\alpha$-WT and mApCaMKII $\alpha$-T286D significantly colocalized with GFP-Shank3-WT in these cells, whereas the colocalization of $\mathrm{mAp}-\mathrm{CaMKII} \alpha$ T286A with GFP-Shank3-WT was much weaker $(p<0.0001)$. The unexpectedly robust colocalization of $\mathrm{mAp}$-CaMKII $\alpha$-WT with GFP-Shanks3-WT may be explained by the observation that $\mathrm{mAp}$-CaMKII $\alpha$-WT is significantly phosphorylated at Thr286 in STH $\mathrm{dh}^{+/+}$cells under these conditions (Fig. 5A, right). Together, these data show that Shank3 colocalization with CaMKII in intact cells is sensitive to Thr286 modification, commensurate with our biochemical data (Fig. 2C).

If colocalization of mAp-CaMKII $\alpha$-WT with GFP-Shank3 requires the direct interaction that we identified in vitro, the RRK/ AAA mutation in the central domain of Shank3 should interfere with colocalization. Therefore, a second series of studies compared the colocalization of mAp-CaMKII $\alpha$-WT with GFP-Shank3-WT or GFP-Shank3-AAA; as an additional negative control, we examined the colocalization of soluble mApple with GFP-Shank3-WT (Fig. 5D). GFP-Shank3-WT and GFP-Shank3-AAA adopted a punctate localization pattern similar to that observed in Figure $5 B$. However, mAp-CaMKII $\alpha$-WT overlapped with GFPShank3-WT punctae, but not GFP-Shank3-AAA (Fig. 5D, compare top to center). The soluble mApple diffusely filled the cell, with minimal overlap with GFP punctae (Fig. $5 D$, bottom). This qualitative assessment was confirmed by image correlation anal- 


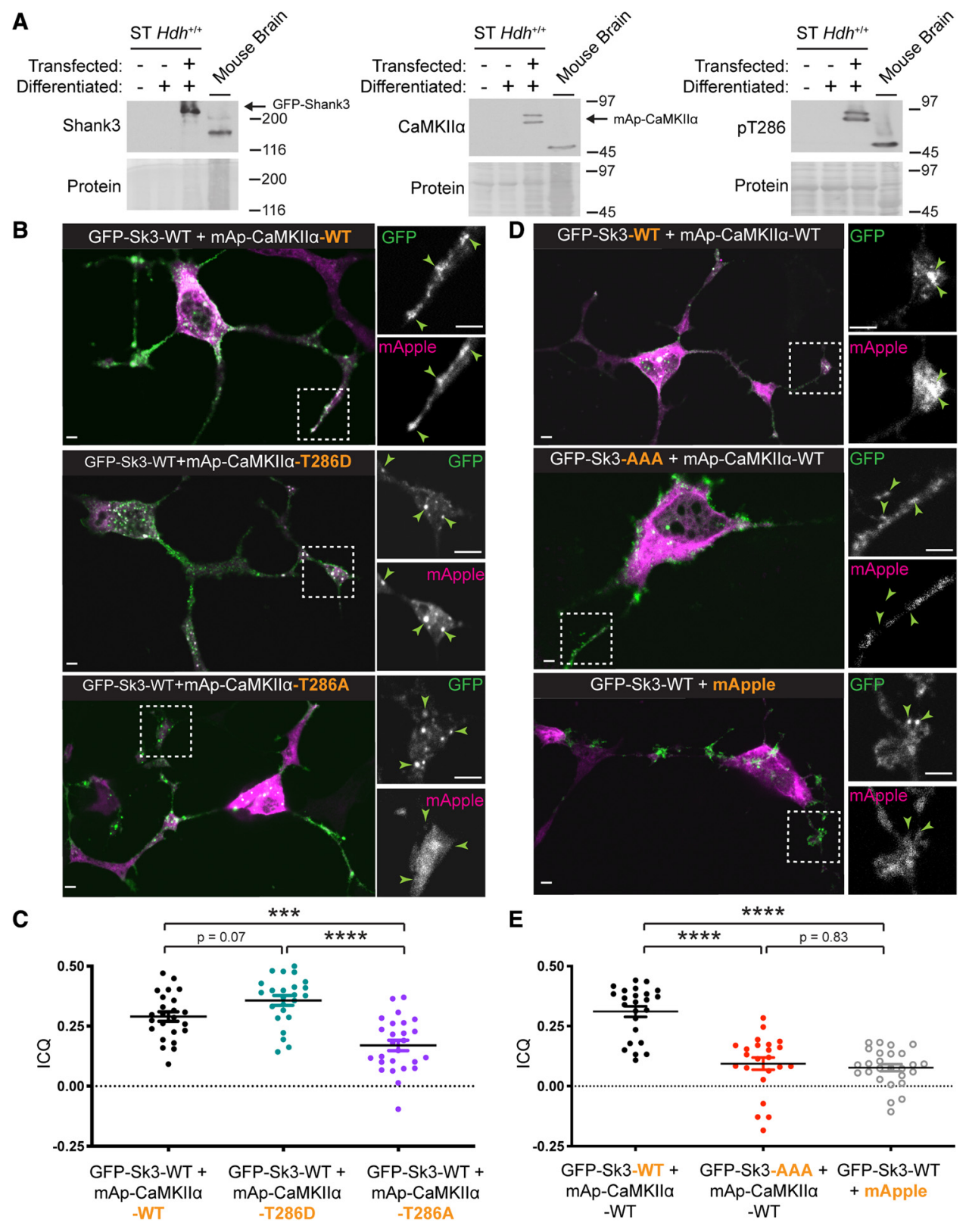

Figure 5. Shank3 ${ }^{949}{ }^{\mathrm{RRK}}{ }^{951}$ to AAA mutation disrupts colocalization of activated CaMKII $\alpha$. A, Immunoblots of undifferentiated, differentiated, and transfected/differentiated STHdh ${ }^{+/+}$cells, with a WT mouse forebrain lysate as positive control. Shank3 and CaMKII $\alpha$ are not expressed in nontransfected STHdh ${ }^{+/+}$cells, and transfected mApple-tagged CaMKII $\alpha$ (mAp-CaMKII $\alpha$ ) is T286-phosphorylated. $\boldsymbol{B}$, Representative images of differentiated STHdh ${ }^{+/+}$cells expressing GFP-Shank3-WT with mAp-CaMKIl $\alpha$-WT (left), mApCaMKII $\alpha$-T286D (middle), or mAp-CaMKII $\alpha$-T286A (right). Inset, Regions (dashed line box) of the processes containing punctate GFP signals (arrowheads) that overlap with mApple signal from CaMKII $\alpha$-WT and-T286D, but not-T286A. C, Intensity correlation analysis quantifying the colocalization of GFP and mAp signals in transfected and differentiated STHdh ${ }^{+/+}$ cells in $\boldsymbol{B}$. Each data point represents an ICQ value from a single cell, with 7-12 cells analyzed from each of three independent cultures/transfections. ICQ values for GFP-Shank3-WT and either mAp-CaMKII $\alpha$-WT (mean \pm SEM: $0.29 \pm 0.02$ ) or mAp-CaMKII $\alpha$-T286D (0.36 \pm 0.02$)$ were significantly more colocalized compared with mAp-CaMKII $\alpha$-T286A (0.17 \pm 0.02$)$ (one-way ANOVA, $F_{(2,71)}=21.35, p<0.0001$ ). Tukey's post hoc test: ${ }^{* * *} p<0.001,{ }^{* * * *} p<0.0001$. D, Representative images of differentiated STHdh ${ }^{+/+}$expressing GFP-Shank3-WT or GFP-Shank3-AAA with soluble mAp or mAp-CaMKII $\alpha$-WT. Inset, Expanded regions of the processes, as in $\boldsymbol{B}$. $\boldsymbol{E}$, Intensity correlation analysis quantifying the colocalization of GFP and mAp signals in transfected and differentiated STHdh ${ }^{+/+}$cells in C. GFP-Shank3-WT and mAp-CaMKIl $\alpha$-WT $(0.31 \pm 0.02)$ are significantly more colocalized than GFP-Shank3-WT and $\mathrm{mAp}(0.07 \pm 0.01)$ or GFP-Shank3-AAA and mAp-CaMKII $\alpha(0.09 \pm 0.02)$ (one-way ANOVA, $\left.F_{(2,70)}=39.55, p<0.0001\right)$. Tukey's post hoc test: ${ }^{* * * *} p<0.0001$. Scale bars: $B, D, 2.5 \mu \mathrm{m}$.

ysis, with ICQ values for overlap of $0.31 \pm 0.02,0.07 \pm 0.01$, and $0.09 \pm 0.02$, respectively (Fig. $5 E$ ). These data indicate that the colocalization of mAp-CaMKII $\alpha$-WT with GFP-Shank3-WT punctae in intact cells is disrupted by the RRK/AAA mutation that we have shown disrupts the direct interaction of CaMKII with Shank3 in vitro.
Effects of Shank3 overexpression on LTCC signaling to the nucleus

Previous studies indicate that Shank3 may be critical for optimal LTCC signaling that increases Ser ${ }^{133}$ phosphorylation of the CREB transcription factor in the nucleus (Zhang et al., 2005). This pathway can be initiated by local $\mathrm{Ca}^{2+}$ influx, without re- 
A
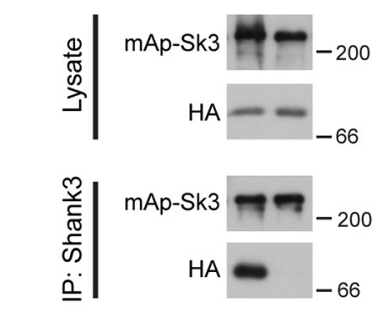

HA-Ca $1.3-C T D: \quad+\quad+$ mAp-Sk3R- ${ }^{\mathrm{R}} \mathrm{PDZ}:-+$ mAp-Sk3R-WT: + -

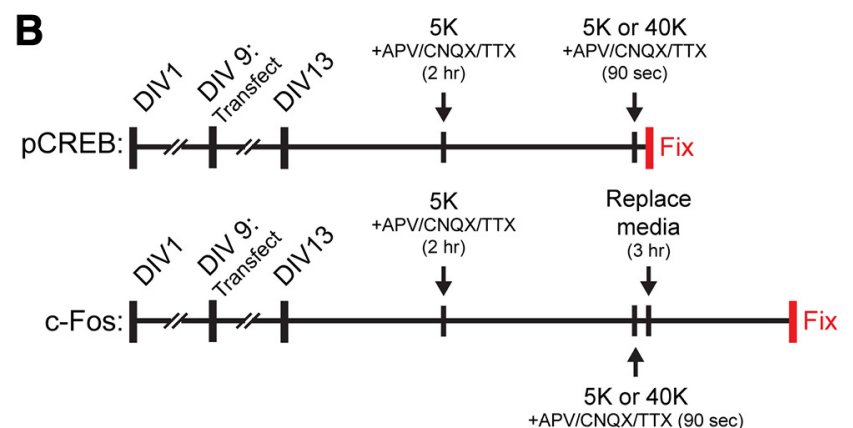

$+\mathrm{APV} / \mathrm{CNQX} / \mathrm{TTX}(90 \mathrm{sec})$
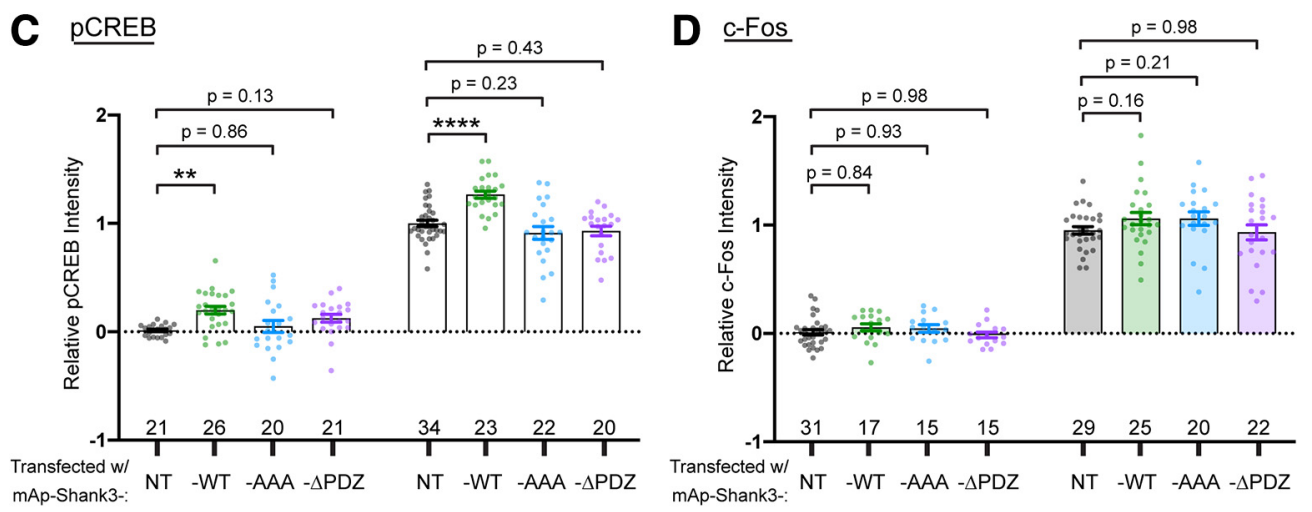

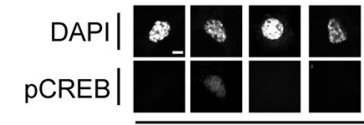

Stimulation:

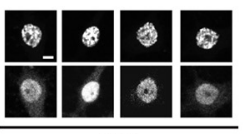

$40 \mathrm{~K}$

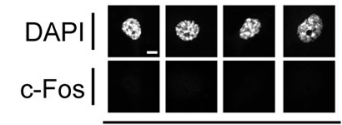

$5 \mathrm{~K}$

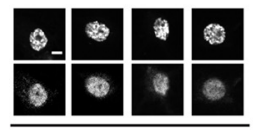

$40 \mathrm{~K}(90 \mathrm{sec})$

Figure 6. Effects of Shank3 overexpression on LTCC signaling to the nucleus. A, Shank3 was immunoprecipitated from soluble fractions of HEK293T cells expressing HA-Ca $1.3-C T D$ with mAp-Shank $3^{R}$-WT or mAp-Shank $3^{R}-\triangle P D Z$. Immunoblots (representative of three biological replicates) demonstrate that HA-Ca 1 1.3-CTD coimmunoprecipitates with mAp-Shank $3^{R}$-WT, but not with mAp-Shank $3^{\mathrm{R}}-\triangle$ PDZ. B, Schematic of experimental protocols. Primary hippocampal neurons were transfected (see below) and then incubated to stimulate LTCC signaling to the nucleus (see Materials and Methods). Neurons were either fixed and stained using DAPI and pSer133-CREB antibodies after a $90 \mathrm{~s}$ depolarization (top: for $C$ ), or incubated for an additional $3 \mathrm{~h}$ in conditioned media before fixation and staining with DAPI and c-Fos antibodies (bottom: for D). C, Overexpression of mAp-Shank3-WT, but not mAp-Shank3-AAA or mAp-Shank3- $\triangle$ PDZ, increases the levels of pCREB staining relative to nontransfected neurons under basal and depolarized conditions (two-way ANOVA with 2 factors [Mutant, Stimulation]: Mutant, $F_{(3,179)}=17.86, p<0.0001 ;$ Stimulation, $F_{(1,179)}$ $=1108, p<0.0001$; Interaction, $\left.F_{(3,179)}=4.442, p<0.01\right)$. Dunnett's multiple-comparisons test: ${ }^{* *} p<0.01$, ${ }^{* * *} p<0.0001$. D, The expression of c-Fos is not affected by overexpression of mAp-Shank3-WT, mAp-Shank3-AAA, or mAp-Shank3- $\Delta$ PDZ (two-way ANOVA with 2 factors [Mutation, Stimulation]: Mutation, $F_{(3,166)}=2.152 ;$ Stimulation, $F_{(1,166)}=830.1, p<0.0001$; Interaction, $F_{(3,166)}=0.3284$, Dunnett's multiple-comparisons test). Error bars indicate mean \pm SEM. Superimposed data points indicate values from single cells accumulated from three to five independent neuronal cultures/transfections. Images below the bar graphs are of representative nuclei for each condition. Scale bars, $5 \mu \mathrm{m}$.

quiring global increases in $\mathrm{Ca}^{2+}$, and requires CaMKII recruitment to this LTCC nanodomain (Wheeler et al., 2008; Ma et al., 2014; Wang et al., 2017). Since Shank3 interacts with several other proteins, in addition to the CTD of $\mathrm{Ca}_{\mathrm{V}} 1.3$ LTCCs (via its PDZ domain) (Zhang et al., 2005) and CaMKII (current observation), any effects of manipulating Shank3 expression on signaling to the nucleus may involve multiple mechanisms. The role of CaMKII binding to Shanks may be revealed by comparing the effects of expressing Shank3-WT and Shank3-AAA (which cannot bind CaMKII). As a complementary tool, we also generated a Shank3 mutant lacking the entire PDZ domain (Shank3- $\triangle$ PDZ) that is unable to interact with the CTD of $\mathrm{Ca}_{\mathrm{V}} 1.3$ (Fig. $6 A$ ). Comparing the effects of expressing Shank3-WT and Shank3- $\Delta$ PDZ in neurons should reveal the role of Shank3 PDZ domain binding to $\mathrm{Ca}_{\mathrm{V}} 1.3$.

We initially compared the effects of overexpressing WT or mutated Shank3 proteins on LTCC/CaMKII-dependent signaling to the nucleus in cultured hippocampal neurons using a wellestablished stimulation paradigm (Fig. 6B, top) (Wheeler et al., 2008; Ma et al., 2014; Li et al., 2016; Wang et al., 2017). Intrinsic neuronal activity was blocked by preincubation in $5 \mathrm{~mm} \mathrm{~K} \mathrm{~K}^{+}$ Tyrode's solution (5K) containing APV and CNQX (to block acti- vation of NMDA- and AMPA-type glutamate receptors, respectively) and TTX (to inhibit voltage-dependent sodium channels). Neurons were then depolarized by replacing the solution with 40 mM K ${ }^{+}$Tyrode's solution (40K), also containing APV, CNQX, and TTX for $90 \mathrm{~s}$. It is well established that this treatment robustly increases nuclear staining detected using a phospho-Ser ${ }^{133}$ specific CREB antibody (pCREB intensity) that can be completely disrupted by the selective LTCC blocker nimodipine (10 $\mu \mathrm{M}$ ) (Wheeler et al., 2012; Wang et al., 2017). To determine whether this depolarization also induces gene expression, the depolarization buffer was replaced with conditioned media after $90 \mathrm{~s}$, and neurons were fixed $3 \mathrm{~h}$ later to immunostain them for expression of the c-Fos immediate early gene (Fig. $6 B$, bottom). The brief neuronal depolarization is sufficient to induce a robust increase in c-Fos protein staining $3 \mathrm{~h}$ later (Fig. $6 D$ ), which can be completely blocked by preincubation with $10 \mu \mathrm{M}$ nimodipine (T.L.P. and R.J.C., unpublished observation).

To identify transfected neurons, Shank3-WT, -AAA, and $-\triangle \mathrm{PDZ}$ were overexpressed as mApple-tagged proteins. Approximately $3 \mathrm{~d}$ after transfection, cultures were fixed under basal (5K) conditions or after the $90 \mathrm{~s} 40 \mathrm{~K}$ depolarization (see above) and examined by confocal microscopy to quantify pCREB signals 
A

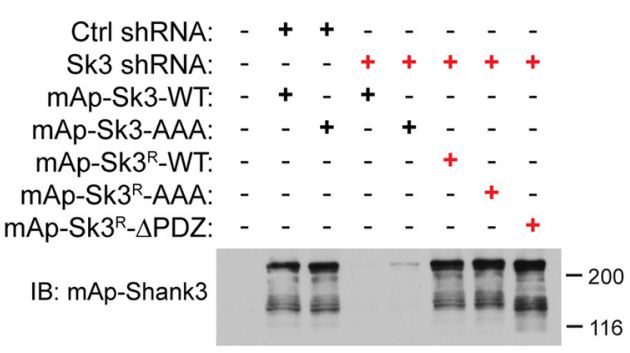

B

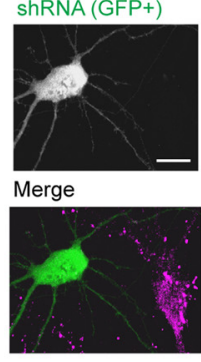

Shank3

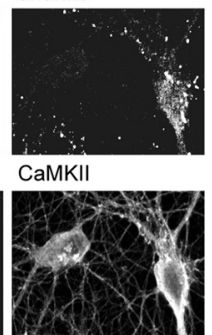

C

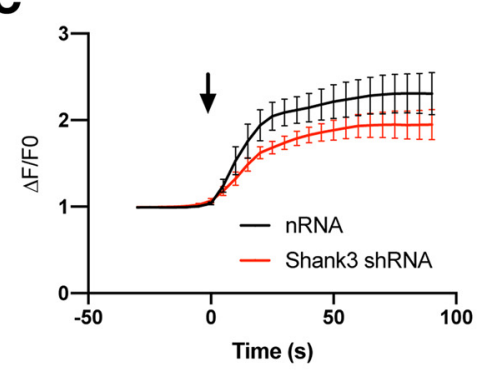

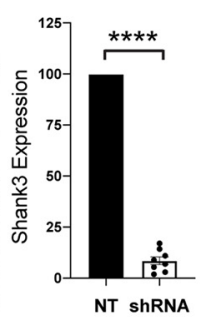

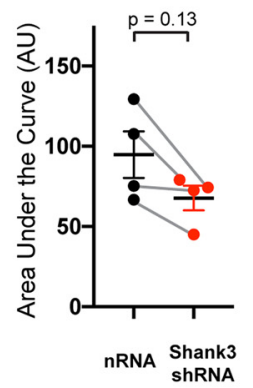

D PCREB
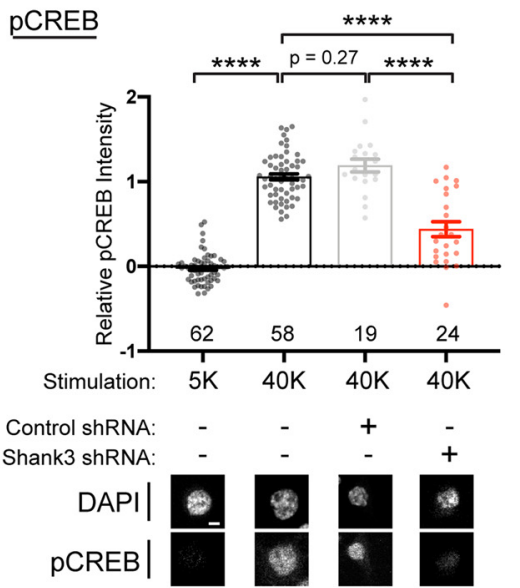

E $\underline{c-F o s}$

$* * *$

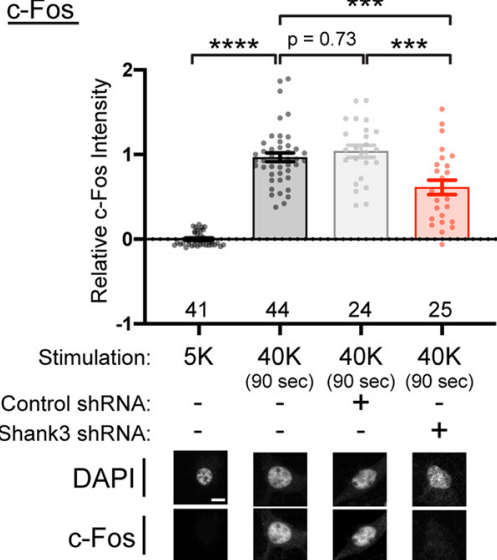

Figure 7. Shank3 knockdown disrupts pCREB signaling and c-Fos expression. $A$, Validation of Shank3 shRNA and mApple-Shank3 shRNA-resistant (mAp-Shank $3^{\mathrm{R}}$ ) expression vectors. Expression of shRNA, mAp-Shank3, and mAp-Shank3 ${ }^{\mathrm{R}}$ in HEK293T cells. Lysates of cells expressing (as indicated above) a control shRNA or Shank3 shRNA, along with mAp-Shank3 constructs with the WT shRNA target sequences (mAp-Shank3-WT and mAp-Shank3-AAA) or contain "silent" mutations that confer shRNA resistance (mAp-Shank $3^{\mathrm{R}}$ constructs) were immunoblotted for Shank3 (NeuroMab antibody). B, DIV13 primary hippocampal neurons transfected at DIV10 with Shank3 shRNA (GFP ${ }^{+}$) and stained for Shank3 (magenta; (ST antibody) and CaMKIl $\alpha$ (white; a marker of excitatory neurons). Neurons expressing the Shank3 shRNA contain substantially reduced levels of Shank3 (reduced by $91 \pm 2 \%$ relative to nearby nontransfected excitatory neurons). ${ }^{* * *} p<0.0001$ (one-sample unpaired Student's $t$ test with equal variance compared with a theoretical value of 100). Error bars indicate mean \pm SEM. Each data point represents a single cell accumulated from three independent neuronal cultures/transfections. Scale bars, $20 \mu \mathrm{m}$. C, Shank3 knockdown has little effect on global $\mathrm{Ca}^{2+}$ influx. Left, fura-2-loaded hippocampal neurons transfected with control shRNA (nRNA) or Shank3 shRNA were equilibrated with Tyrode's solution containing $5 \mathrm{~mm} \mathrm{KCl} \mathrm{for} \mathrm{3-5} \mathrm{min} \mathrm{and} \mathrm{switched} \mathrm{(black} \mathrm{arrow)} \mathrm{to} \mathrm{Tyrode's} \mathrm{solution} \mathrm{containing} 40 \mathrm{~mm}$ KCl for $90 \mathrm{~s}$. The graph plots mean $\pm \mathrm{SEM} \triangle \mathrm{F} / \mathrm{F} 0$ values for the last $30 \mathrm{~s}$ of the equilibration period and during depolarization from four independent experiments (12-90 cells per replicate). The data were analyzed using a two-way repeated-measures ANOVA: Factor 1 (time), $F_{(1.342,8.049)}=32.63, p=0.0003 ;$ Factor 2 (control/shRNA), $F_{(1,6)}=1.703, p=0.2398 ;$ Interaction, $F_{(35,210)}=0.8281, p=0.7423$. Right, Comparison of average areas under the curve from each independent experiment revealed no statistically significant difference $(n=4, p=0.13$; paired Student's $t$ test with equal variance). $\boldsymbol{D}$, The robust increase in pCREB levels following a brief depolarization (as in Fig. 6A) is significantly reduced in cells expressing the Shank3 shRNA (red bar), but not control shRNA (gray bar) (5K vs 40K: unpaired Student's $t$ test with equal variance, ${ }^{* * * *} p<0.0001 ; 40 \mathrm{~K}$ stimulations: one-way ANOVA, $F_{(2,98)}=38.17, p<0.0001$; Tukey's post hoc test, $\left.{ }^{* * * *} p<0.0001\right)$. E, Similarly, the robust increase in c-Fos expression $3 \mathrm{~h}$ after the brief depolarization is significantly reduced in cells expressing Shank3 shRNA (red bar), but not control shRNA (gray bar) (5K vs 40K: unpaired Student's t test with equal variance, ${ }^{* * * *} p<0.0001 ; 40 \mathrm{~K}$ stimulations: one-way ANOVA, $F_{(2,90)}=9.990, p<0.001$, Tukey's post hoc test, $\left.{ }^{* * *} p<0.001\right)$. Error bars indicate mean \pm SEM. Each data point represents a single cell accumulated from three independent neuronal cultures/transfections. Images below the bar graphs are of representative nuclei for each condition. Scale bars, $5 \mu \mathrm{m}$.

in DAPI-stained nuclei of mApple- and CaMKII $\alpha$-expressing neurons relative to CaMKII $\alpha$-expressing neurons in nontransfected cultures. The overexpression of mAp-Shank3-WT resulted in a small but significant increase in PCREB intensity in both unstimulated and depolarized neurons relative to nontransfected neurons (Fig. 6C, compare green and black bars). Notably, this increase of pCREB intensity was not detected in neurons overexpressing similar levels of mAp-Shank3-AAA or mAp-Shank3- $\triangle \mathrm{PDZ}$ (Fig. 6C, blue and purple bars). However, the modest increases in PCREB intensity following overexpression of mAp-Shank3-WT were not sufficient to enhance c-Fos expression in unstimulated or depolarized neurons (Fig. 6D). Together, these data indicate that, under these conditions, the overexpression of Shank 3 has relatively limited effects on LTCC signaling to the nucleus, perhaps because these cells contain significant levels of endogenous Shank3.

\section{Shank3 is required for LTCC-dependent CREB} phosphorylation and gene expression

To further investigate the role of Shank 3 in LTCC signaling to the nucleus, we adopted an shRNA knockdown approach to suppress the expression of endogenous Shank3. First, we confirmed that a previously used shRNA targeting Shank3 (Verpelli et al., 2011) essentially completely suppressed the expression of mAp-Shank3WT or mAp-Shank3-AAA in HEK293 cells, whereas a control shRNA (Boudkkazi et al., 2014) has no effect (Fig. 7A, lanes 2 and 3). Moreover, the Shank 3 shRNA has no effect on expression of an shRNA-resistant forms of Shank3 (mAp-Shank $3^{\mathrm{R}}$ ) (Fig. $7 \mathrm{~A}$, lanes 6-8). Furthermore, staining for Shank3 protein in CaMKIIpositive primary hippocampal neurons was reliably decreased in neurons expressing the Shank3 shRNA relative to nearby non- 
A pCREB

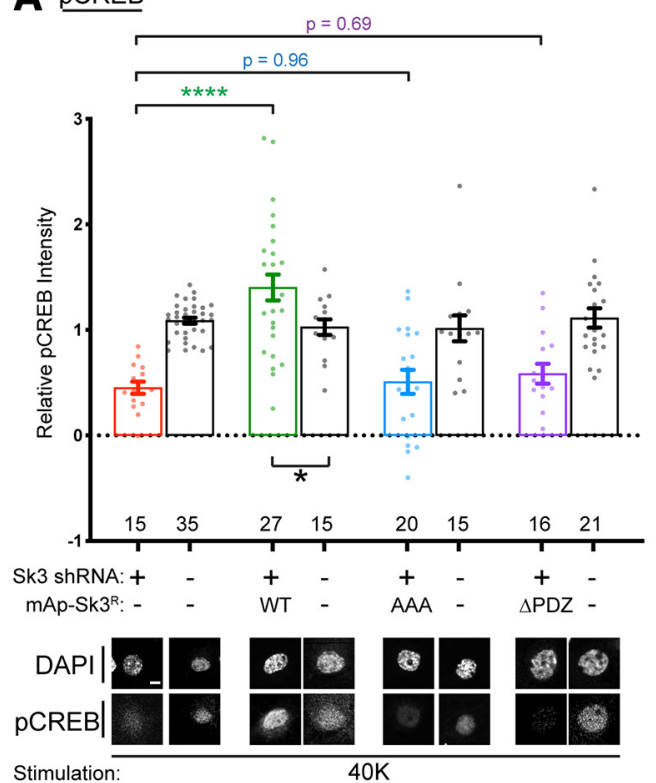

B $\stackrel{\text { c-Fos }}{ }$

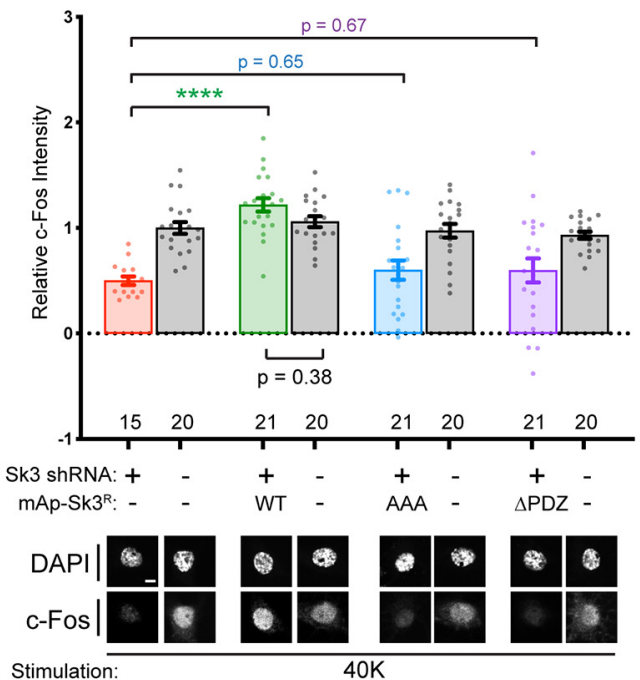

Figure 8. Rescue of pCREB signaling and c-Fos expression after Shank3 shRNA knockdown. The Shank3 shRNA construct was transfected alone or with shRNA-resistant mAp-Shank $3^{\mathrm{R}}$-WT (green bar), $m A$ p-Shank $3^{R}$-AAA (blue bar), or mAp-Shank3 ${ }^{R}-\triangle P D Z$ (purple bar). Neurons were depolarized for 90 s, and the levels of $p C R E B(\boldsymbol{A})$ and $c-F 0 s(\boldsymbol{B})$ were determined (see Materials and Methods) in transfected (colored bars) and nearby nontransfected (black bars) neurons. $A$, The expression of mAp-Shank $3^{R}$-WT, but not mAp-Shank $3^{R}$-AAA or mAp-Shank $3^{R}-\triangle P D Z$, rescued signaling to pCREB relative to shRNA alone. In addition, neurons expressing Shank3-WT had significantly higher pCREB signal relative to nearby, nontransfected neurons (tw0-way ANOVA with 2 factors [Mutant, Transfection]: Mutant, $F_{(3,156)}=10.14, p<0.0001$; Transfection, $F_{(1,156)}=22.70, p<0.0001$; Interaction, $F_{(3,156)}=12.29, p<0.0001$, comparison between mutants: Dunnett's multiplecomparisons test, ${ }^{* * * *} p<0.0001$; comparison between transfected/nontransfected cells: Sidak's multiple-comparisons test, $\left.{ }^{*} p<0.05\right)$. $\boldsymbol{B}$, The expression of mAp-Shank ${ }^{\mathrm{R}}$-WT, but not mAp-Shank $3^{\mathrm{R}}$-AAA or mAp-Shank3 ${ }^{\mathrm{R}}$ - $\Delta$ PDZ, rescued signaling to increase c-Fos expression relative to shRNA alone (two-way ANOVA with 2 factors ([Mutant, Transfection]: Mutant, $F_{(3,150)}=$ $13.80, p<0.0001$; Transfection, $F_{(1,150)}=26.52, p<0.0001$; Interaction, $F_{(3,150)}=8.149, p<0.0001$, comparison between mutants: Dunnett's multiple-comparisons test, ${ }^{* * * *} p<0.0001$; comparison between transfected/nontransfected cells: Sidak's multiple-comparisons test). Error bars indicate mean \pm SEM. Each data point represents a single cell, accumulated from four or five independent neuronal cultures/transfections. Images below the bar graphs are of representative nuclei for each condition. Scale bars, $5 \mu \mathrm{m}$.

transfected neurons (Fig. 7B). Thus, this shRNA effectively suppresses Shank3 expression in neurons.

Deletion of five C-terminal amino acids from $\mathrm{Ca}_{\mathrm{V}} 1.3$ prevents binding to Shank3 but has little impact on LTCC gating, or on whole-cell LTCC current amplitude or current-voltage relationship in heterologous cells and in cultured neurons, or on total $\mathrm{Ca}_{\mathrm{V}}$ 1.3-LTCC-mediated neuronal $\mathrm{Ca}^{2+}$ influx (Zhang et al., 2005). However, the impact of neuronal Shank 3 knockdown on LTCC-mediated $\mathrm{Ca}^{2+}$ influx has not been determined. Therefore, we used fura- 2 to assess somatic $\mathrm{Ca}^{2+}$ responses during a $90 \mathrm{~s}, 40 \mathrm{~K}$ depolarization as used for studying E-T coupling (see above). Comparison of average $\mathrm{Ca}^{2+}$ responses $(\Delta \mathrm{F} / \mathrm{F} 0)$ over time in neurons expressing control or Shank 3 shRNA from four independent experiments revealed no statistically significant difference (Fig. 7C, left). Similarly, Shank3 knockdown had no statistically significant impact on total $\mathrm{Ca}^{2+}$ influx, as estimated from average areas under the curve from each experiment (Fig. $7 C$, right), However, there was a trend for a small reduction of $\mathrm{Ca}^{2+}$ influx in Shank3 shRNA neurons. Together, these data indicate that knockdown of Shank3 expression results in little effect on global $\mathrm{Ca}^{2+}$ influx via LTCCs under these conditions.

Neuronal cultures were then transfected to express either control or Shank3 shRNA and depolarized for $90 \mathrm{~s}$. While control shRNA had no effect on pCREB intensity (Fig. $7 D$, gray bar), neurons expressing Shank3 shRNA had a significant, if partial, reduction in PCREB intensity relative to nontransfected neurons and control shRNA-expressing neurons (Fig. $7 D$, red bar). This decrease in pCREB intensity in neurons expressing the Shank3 shRNA was mirrored by a parallel decrease of c-Fos expression when assessed $3 \mathrm{~h}$ after the brief depolarization
(Fig. 7E, red bar), whereas c-Fos was robustly expressed in nontransfected neurons and neurons expressing control shRNA $3 \mathrm{~h}$ after stimulation. Thus, Shank 3 expression appears to be necessary for the full extent of both CREB phosphorylation as well as c-Fos expression following a brief neuronal depolarization.

\section{Rescue of shRNA effects by Shank3-WT, but not Shank3-AAA or Shank3- $\triangle$ PDZ}

We then investigated whether Shank3 shRNA-induced suppression of E-T coupling could be rescued by Shank 3 reexpression from shRNA-resistant constructs $\left(\mathrm{mAp}-\mathrm{Shank} 3^{\mathrm{R}}\right)$. Neurons were transfected to express Shank3 shRNA alone or Shank3 shRNA plus either mAp-Shank $3^{R}$-WT, mAp-Shank $3^{\mathrm{R}}$-AAA, or mAp-Shank $3^{R}-\triangle P D Z$. Following depolarization, pCREB staining was quantified in neurons expressing the shRNA, and in nearby nontransfected neurons within the same dish, to provide an internal control for each experiment with a similar robust increase of pCREB staining intensity under all conditions (Fig. $8 A$, black bars). As seen in Figure 7D, Shank3 shRNA expression significantly, if partially, reduced the pCREB signal (Fig. $8 A$, red bar), but expression of $\mathrm{mAp}$-Shank $3^{\mathrm{R}}$-WT rescued pCREB intensity to a level slightly but significantly higher than that in nontransfected neurons (Fig. 8A, green bar). However, the level of pCREB staining was not rescued by expression of either mAp-Shank $3^{\mathrm{R}}$-AAA or mAp-Shank $3^{\mathrm{R}}$ - $\triangle \mathrm{PDZ}$ (Fig. 8 A, blue and purple bars). Moreover, analysis of parallel neuronal cultures $3 \mathrm{~h}$ following the brief depolarization revealed that partial suppression of c-Fos protein expression by the Shank 3 shRNA was rescued by $\mathrm{mAp}$-Shank $3^{\mathrm{R}}$-WT, but not by $\mathrm{mAp}$-Shank $3^{\mathrm{R}}$ - 
AAA or mAp-Shank $3^{\mathrm{R}}-\Delta \mathrm{PDZ}$ (Fig. $8 B$ ). However, c-Fos expression in neurons expressing both the Shank3 shRNA and mAp-Shank $3^{\mathrm{R}}$-WT was not significantly different from c-Fos levels in nearby nontransfected neurons. Together, these findings significantly extend previous studies by showing that the Shank3 PDZ domain is important for LTCC signaling to the nucleus, presumably by binding to $\mathrm{Ca}_{\mathrm{V}} 1.3$ (Zhang et al., 2005), and that CaMKII binding to Shank3 also plays a key role in this pathway. Importantly, these interactions with Shank3 play a key role not only in depolarization-induced increases of CREB phosphorylation, but also for the downstream increase in expression of c-Fos, an important immediate early gene.

\section{Discussion}

We recently used a proteomics approach to show that synaptic CaMKII complexes immunoprecipitated from mouse forebrain contain many proteins, including substantial amounts of Shank3 (Baucum et al., 2015). In addition, we reported that Shank3CaMKII complexes can be coimmunoprecipitated from HEK293T cells overexpressing Shank3 and CaMKII (Stephenson et al., 2017). However, coimmunoprecipitation of two proteins may arise from direct or indirect interactions of the proteins involved. Here, we explicitly demonstrate a direct interaction between purified Shank3 and purified CaMKII and define its regulation by CaMKII activation. Thr286-autophosphorylated CaMKII $\alpha$ robustly and directly binds to a central domain in Shank3 with no previously defined biochemical or physiological function, and mutation of three basic residues within this domain to Ala (AAA mutation) largely prevents binding. In vitro, this Shank3 domain binds much more weakly to CaMKII $\alpha$ activated only by $\mathrm{Ca}^{2+}$ / CaM binding. The colocalization of CaMKII with Shank3 in intact cells is regulated by CaMKII activation and disrupted by the AAA mutation, concordant with our in vitro findings. Moreover, robust coimmunoprecipitation of CaMKII $\alpha$ with Shank3 from mouse forebrain extracts is largely abrogated by a knock-in Thr286 to Ala mutation, showing that CaMKII $\alpha$ Thr286 autophosphorylation is required for robust CaMKII-Shank3 interactions in vivo. From a functional perspective, we show that Shank3 expression is important for normal LTCC-dependent E-T coupling in cultured hippocampal neurons, as measured by CREB phosphorylation and the expression of c-Fos, a critical immediate early gene. Finally, our Shank3 shRNA rescue studies using WT and mutated Shank3 proteins indicate that interactions with both CaMKII and the $\mathrm{Ca}_{\mathrm{V}} 1.3$ LTCC are important for normal E-T coupling. Together, these data substantially advance our understanding of the molecular mechanisms underlying a novel biological role for a major synaptic scaffolding protein.

CaMKII has diverse physiological functions, and the present findings add to a growing body of evidence that specific functions of CaMKII are controlled in part by interactions with CaMKAPs. Like Shank3, most CaMKAPs preferentially bind activated CaMKII conformations, induced by either $\mathrm{Ca}^{2+} / \mathrm{CaM}$ binding and/or Thr286 autophosphorylation. Therefore, CaMKII activation acts as a switch to stimulate binding to Shank3 and other CaMKAPs. Interestingly, Shank3 and the GluN2B subunit of the NMDAR exhibit different selectivity for distinct activated CaMKII conformations. $\mathrm{Ca}^{2+} / \mathrm{CaM}$ binding alone is insufficient for GluN2B binding but partially supports interactions with Shank3 and densin, relative to Thr286-autophosphorylated CaMKII $\alpha$ (Robison et al., 2005a; Jiao et al., 2011). While the physiological significance of these differences is unclear, they presumably reflect subtle variations in how CaMKAPs interact with CaMKII $\alpha$. Consistent with this idea, the CaMKII binding domains of activation-dependent
CaMKAPs can be subclassified based on sequence homology. CaMKII binding domains in GluN2B subunits and LTCC $\beta 1 / 2$ subunits share sequence homology with the CaMKII $\alpha$ autoregulatory domain, surrounding the Thr286 autophosphorylation site (Strack et al., 2000; Grueter et al., 2008). However, the internal CaMKII binding domain in densin, another synaptic scaffolding protein, has a distinct amino acid sequence, resembling that of the naturally occurring CaMKII inhibitor protein CaMKIIN (Jiao et al., 2011). Furthermore, recently identified CaMKII binding domains in LTCC $\mathrm{Ca}_{\mathrm{V}} 1.2$ and $\mathrm{Ca}_{\mathrm{V}} 1.3 \alpha 1$ subunits and the mGlu5 metabotropic glutamate receptor contain critical tribasic residue motifs (Wang et al., 2017; Marks et al., 2018), similar to that in Shank3 identified here (Fig. 3). However, the tribasic residue motif alone is insufficient for CaMKII binding because Thr286-autophosphorylated CaMKII $\alpha$ does not bind two GSTShank3 proteins (\#3 and \#4a) that contain tribasic sequences $\left({ }^{707}\right.$ Arg-Arg-Lys ${ }^{709}$ and ${ }^{919}$ Lys-Arg-Arg ${ }^{921}$, respectively) (Figs. 2B, $3 B)$. Further studies will be needed to explore contributions of residues surrounding the tribasic binding motifs, and perhaps secondary or tertiary structure, to CaMKII binding with these CaMKAPs.

It is well established that activated CaMKII and Shank3 are highly enriched in dendritic spines (Shen and Meyer, 1999; Boeckers et al., 2005), and are also found in complexes containing NMDARs and other CaMKAPs (Naisbitt et al., 1999; Baucum et al., 2015). Since dendritic spines contain several CaMKAPs, it can be difficult to discern their individual contributions to modulating CaMKII localization and function. However, knock-in mutations of GluN2B in mice that block CaMKII binding to GluN2B and reduce the amount of CaMKII in postsynaptic densities also impair both synaptic plasticity and memory recall (Halt et al., 2012). While Shank3 knockdown has been shown to disrupt NMDAR-mediated postsynaptic currents and DHPG-induced LTD (Verpelli et al., 2011; Duffney et al., 2013), roles of specific protein interactions with Shank3 are poorly understood. We posit that one function of Shank3 is to target a subpopulation of CaMKII holoenzymes to postsynaptic LTCC complexes.

Our studies show that Shank3 has important roles in E-T coupling initiated by LTCC-dependent $\mathrm{Ca}^{2+}$ influx and CaMKII activation to stimulate CREB phosphorylation and c-Fos expression. Precise regulation of gene transcription is important for long-term, activity-dependent changes in synaptic properties and behavior. Diverse stimulation paradigms increase Ser ${ }^{133}$ phosphorylation of the nuclear CREB transcription factor to stimulate immediate early gene transcription, and CREB is important for learning and long-term memory (for review, see Shaywitz and Greenberg, 1999; Alberini, 2009; Kandel, 2012; Kida and Serita, 2014). Plasma membrane LTCCs are major regulators of nuclear CREB phosphorylation and immediate early gene expression following depolarization (Dolmetsch, 2003; Flavell and Greenberg, 2008; Wheeler et al., 2008; Bading, 2013). Both major neuronal LTCC $\alpha 1$ subunits, $\mathrm{Ca}_{\mathrm{V}} 1.2$ and $\mathrm{Ca}_{\mathrm{V}} 1.3$, can initiate LTCC-CREB signaling, depending on the brain region (Hetzenauer et al., 2006) and the strength of depolarization (Zhang et al., 2006). The CTDs of $\mathrm{Ca}_{\mathrm{V}} 1.2$ and $\mathrm{Ca}_{\mathrm{V}} 1.3$ contain canonical binding motifs for Class 1 PDZ domains, and deletion of these motifs disrupts channel trafficking and clustering, as well as downstream signaling to increase CREB phosphorylation, although these mutations do not appear to affect global increases in $\mathrm{Ca}^{2+}$ following neuronal depolarization (Weick et al., 2003; Zhang et al., 2005). The CTD mutations might disrupt CREB signaling by interfering with the local LTCC nanodomain and/or with conformational changes in LTCCs that are required for E-T 
coupling (Li et al., 2016). However, CTDs of $\mathrm{Ca}_{\mathrm{V}} 1.2$ and $\mathrm{Ca}_{\mathrm{V}} 1.3$ interact with distinct proteins. The CTD of $\mathrm{Ca}_{\mathrm{V}} 1.3$, but not $\mathrm{Ca}_{\mathrm{V}} 1.2$, can interact with PDZ domains of Shank1 or Shank3 (Zhang et al., 2005), but the roles of Shanks in LTCC-CREB signaling have not been previously investigated. Our shRNA experiments showed that Shank3 expression is essential for maximal LTCC-CREB-c-Fos signaling, with little impact on global $\mathrm{Ca}^{2+}$ signals. Moreover, shRNA rescue experiments revealed that the Shank3 PDZ domain is required for E-T coupling, consistent with the idea that Shank3 PDZ domain binding to the $\mathrm{Ca}_{\mathrm{V}} 1.3$ CTD is required, and significantly extending prior findings. Residual E-T coupling following Shank3 knockdown may be mediated by Shank3-independent $\mathrm{Ca}_{\mathrm{v}} 1.2$ LTCC signaling, although our data cannot preclude contributions from low levels of residual Shank3 expression or from Shank1. Nevertheless, our observations strongly support a model in which Shank3 actions within the LTCC/ $\mathrm{Ca}^{2+}$ nanodomain are required for efficient E-T coupling to increase both CREB phosphorylation and c-Fos expression.

The form of LTCC-dependent E-T coupling studied herein also requires direct interaction of CaMKII with the N-terminal domain of $\mathrm{Ca}_{\mathrm{V}} 1.3 \alpha 1$ subunits in LTCC nanodomains (Wheeler et al., 2008; Wang et al., 2017). How can this observation be reconciled with the current finding that mutation of the tribasic residue motif in Shank3 to prevent CaMKII binding also disrupts E-T coupling? Since CaMKII holoenzymes can bind simultaneously to multiple CaMKAPs (Robison et al., 2005b), different subunits within a single dodecameric CaMKII holoenzyme may interact with $\mathrm{Ca}_{\mathrm{V}} 1.3$ and Shank3, potentially providing a conformational constraint on LTCC cytoplasmic domains that could affect $\mathrm{Ca}^{2+}$ influx. Alternatively, $\mathrm{Ca}_{\mathrm{V}} 1.3$ and Shank 3 may recruit two different CaMKII holoenzymes to the LTCC nanodomain to facilitate a trans-holoenzyme autophosphorylation that appears to be required for shuttling $\mathrm{Ca}^{2+} / \mathrm{CaM}$ to the nucleus to stimulate CREB phosphorylation (Ma et al., 2014; Cohen et al., 2018). Both hypotheses predict that loss of any one of these three proteins or disruption of any one of their mutual interactions would interfere with this form of E-T coupling.

Although mutual interactions of CaMKII, Shank 3 , and $\mathrm{Ca}_{\mathrm{V}} 1.3$ LTCCs are required to initiate this form of E-T coupling, the precise role of these interactions remains unresolved. Knockdown of CaMKII or Shank3 expression has little effect on depolarizationinduced global $\mathrm{Ca}^{2+}$ signals under our conditions (Fig. 7C) (Wang et al., 2017). However, it remains possible that CaMKII and/or Shank3 modulate $\mathrm{Ca}^{2+}$ concentrations within the LTCC nanodomain. Although CaMKII can potentiate net $\mathrm{Ca}^{2+}$ influx via $\mathrm{Ca}_{\mathrm{V}} 1.3$ LTCCs by decreasing $\mathrm{Ca}^{2+}$-dependent inactivation in heterologous cells (Jenkins et al., 2010), this modulation requires coexpression of densin, another synaptic CaMKAP containing a PDZ domain that binds to the $\mathrm{Ca}_{V} 1.3$ CTD. Like Shank3, densin has no direct effect on the biophysical properties of LTCCs (Zhang et al., 2005; Jenkins et al., 2010), but Shank3 presumably competes with densin for binding to the CTD of $\mathrm{Ca}_{\mathrm{V}} 1.3$. Shank3 com-

petition might be predicted to disrupt CaMKII- and densindependent facilitation of $\mathrm{Ca}^{2+}$ influx via $\mathrm{Ca}_{\mathrm{V}} 1.3$, reducing overall $\mathrm{Ca}^{2+}$ influx, and it is unclear how this could enhance the initiation of E-T coupling. However, it is possible that Shank3 supports CaMKII modulation of $\mathrm{Ca}_{\mathrm{V}} 1.3$ inactivation in a similar (or perhaps distinct) manner. CaMKII also was reported to mediate the effects of IGF1 to facilitate $\mathrm{Ca}_{\mathrm{V}} 1.3$ currents and CREB phosphorylation at weaker depolarizing membrane potentials (Gao et al., 2006). Specific mechanisms underlying both of these effects of CaMKII on $\mathrm{Ca}_{\mathrm{v}} 1.3$ remain poorly understood, but it is possible that CaMKII phosphorylation of $\mathrm{Ca}_{\mathrm{V}} 1.3 \alpha 1$ or $\beta$ subunits or Shank3 is involved. A significant challenge in elucidating these mechanisms will be to assess their impact within the specific context of the LTCC/Ca ${ }^{2+}$ nanodomain that initiates E-T coupling. Clearly, further studies will be needed to more precisely define these biochemical mechanisms.

SHANK3 mutations are strongly linked to ASD and other neuropsychiatric disorders (Gauthier et al., 2010; Herbert, 2011), and c-Fos expression is dysregulated in rodent ASD models (Orlandini et al., 1996; Williams and Umemori, 2014; Dubiel and Kulesza, 2015). Increases or decreases in Shank3 expression may be associated with distinct neuropsychiatric phenotypes (Bozdagi et al., 2010; Han et al., 2013; Uchino and Waga, 2013). Moreover, gain-of-function $\mathrm{Ca}_{\mathrm{V}} 1.3$ mutations have been identified in patients with ASD (Pinggera et al., 2015, 2017) and a $\mathrm{Ca}_{\mathrm{V}} 1.2$ a point mutation associated with Timothy syndrome increases CREB phosphorylation (Li et al., 2016), perhaps indicating that hyperphosphorylation of CREB may be involved in some neuropsychiatric disorders. Diverse changes in E-T coupling may result from disruption of the LTCC nanodomain due to altered interactions between ASD-linked postsynaptic proteins (Bourgeron, 2009). Our recent work showed that an ASD-linked de novo CaMKII $\alpha$ E183V mutation disrupts interactions with Shank3 and several other CaMKAPs (Stephenson et al., 2017), including the $\mathrm{Ca}_{\mathrm{V}} 1.3$ NTD (T.L.P., J.R.S., and R.J.C., unpublished observations). Thus, even though initial studies failed to detect gross changes in CaMKII expression following genetic disruptions of Shank3 (e.g., Peça et al., 2011), the present findings suggest that more detailed investigations of the role of CaMKII in animal models of neuropsychiatric disorders are warranted.

\section{References}

Akita T, Aoto K, Kato M, Shiina M, Mutoh H, Nakashima M, Kuki I, Okazaki S, Magara S, Shiihara T, Yokochi K, Aiba K, Tohyama J, Ohba C, Miyatake S, Miyake N, Ogata K, Fukuda A, Matsumoto N, Saitsu H (2018) De novo variants in CAMK2A and CAMK2B cause neurodevelopmental disorders. Ann Clin Transl Neurol 5:280-296.

Alberini CM (2009) Transcription factors in long-term memory and synaptic plasticity. Physiol Rev 89:121-145.

Bading H (2013) Nuclear calcium signalling in the regulation of brain function. Nat Rev Neurosci 14:593-608.

Baucum AJ 2nd, Jalan-Sakrikar N, Jiao Y, Gustin RM, Carmody LC, Tabb DL, Ham AJ, Colbran RJ (2010) Identification and validation of novel spinophilin-associated proteins in rodent striatum using an enhanced ex vivo shotgun proteomics approach. Mol Cell Proteomics 9:1243-1259.

Baucum AJ 2nd, Shonesy BC, Rose KL, Colbran RJ (2015) Quantitative proteomics analysis of CaMKII phosphorylation and the CaMKII interactome in the mouse forebrain. ACS Chem Neurosci 6:615-631.

Bayer KU, De Koninck P, Leonard AS, Hell JW, Schulman H (2001) Interaction with the NMDA receptor locks CaMKII in an active conformation. Nature 411:801-805.

Bayer KU, LeBel E, McDonald GL, O’Leary H, Schulman H, De Koninck P (2006) Transition from reversible to persistent binding of CaMKII to postsynaptic sites and NR2B. J Neurosci 26:1164-1174.

Benito E, Valor LM, Jimenez-Minchan M, Huber W, Barco A (2011) cAMP response element binding protein is a primary hub of activity-driven neuronal gene expression. J Neurosci 31:18237-18250.

Boeckers TM, Liedtke T, Spilker C, Dresbach T, Bockmann J, Kreutz MR, Gundelfinger ED (2005) C-terminal synaptic targeting elements for postsynaptic density proteins ProSAP1/Shank2 and ProSAP2/Shank3. J Neurochem 92:519-524.

Boudkkazi S, Brechet A, Schwenk J, Fakler B (2014) Cornichon2 dictates the time course of excitatory transmission at individual hippocampal synapses. Neuron 82:848-858.

Bourgeron T (2009) A synaptic trek to autism. Curr Opin Neurobiol 19: 231-234.

Bozdagi O, Sakurai T, Papapetrou D, Wang X, Dickstein DL, Takahashi N, Kajiwara Y, Yang M, Katz AM, Scattoni ML, Harris MJ, Saxena R, Silver- 
man JL, Crawley JN, Zhou Q, Hof PR, Buxbaum JD (2010) Haploinsufficiency of the autism-associated Shank3 gene leads to deficits in synaptic function, social interaction, and social communication. Mol Autism 1:15.

Chia PH, Zhong FL, Niwa S, Bonnard C, Utami KH, Zeng R, Lee H, Eskin A, Nelson SF, Xie WH, Al-Tawalbeh S, El-Khateeb M, Shboul M, Pouladi MA, Al-Raqad M, Reversade B (2018) A homozygous loss-of-function CAMK2A mutation causes growth delay, frequent seizures and severe intellectual disability. Elife 7:e32451.

Cohen SM, Suutari B, He X, Wang Y, Sanchez S, Tirko NN, Mandelberg NJ, Mullins C, Zhou G, Wang S, Kats I, Salah A, Tsien RW, Ma H (2018) Calmodulin shuttling mediates cytonuclear signaling to trigger experiencedependent transcription and memory. Nat Commun 9:2451.

Dick IE, Joshi-Mukherjee R, Yang W, Yue DT (2016) Arrhythmogenesis in Timothy syndrome is associated with defects in $\mathrm{Ca}(2+)$-dependent inactivation. Nat Commun 7:10370.

Dittgen T, Nimmerjahn A, Komai S, Licznerski P, Waters J, Margrie TW, Helmchen F, Denk W, Brecht M, Osten P (2004) Lentivirus-based genetic manipulations of cortical neurons and their optical and electrophysiological monitoring in vivo. Proc Natl Acad Sci U S A 101:18206-18211.

Dolmetsch R (2003) Excitation-transcription coupling: signaling by ion channels to the nucleus. Sci STKE 2003:PE4.

Dubiel A, Kulesza RJ (2015) Prenatal valproic acid exposure disrupts tonotopic c-fos expression in the rat brainstem. Neuroscience 311:349-361.

Duffney LJ, Wei J, Cheng J, Liu W, Smith KR, Kittler JT, Yan Z (2013) Shank3 deficiency induces NMDA receptor hypofunction via an actindependent mechanism. J Neurosci 33:15767-15778.

Ebert DH, Greenberg ME (2013) Activity-dependent neuronal signalling and autism spectrum disorder. Nature 493:327-337.

Flavell SW, Greenberg ME (2008) Signaling mechanisms linking neuronal activity to gene expression and plasticity of the nervous system. Annu Rev Neurosci 31:563-590.

Gallo FT, Katche C, Morici JF, Medina JH, Weisstaub NV (2018) Immediate early genes, memory and psychiatric disorders: focus on c-Fos, Egr1 and Arc. Front Behav Neurosci 12:79.

Gao L, Blair LA, Salinas GD, Needleman LA, Marshall J (2006) Insulin-like growth factor-1 modulation of CaV1.3 calcium channels depends on $\mathrm{Ca}^{2+}$ release from IP3-sensitive stores and calcium/calmodulin kinase II phosphorylation of the alpha1 subunit EF hand. J Neurosci 26:6259-6268.

Gauthier J, Champagne N, Lafrenière RG, Xiong L, Spiegelman D, Brustein E, Lapointe M, Peng H, Côté M, Noreau A, Hamdan FF, Addington AM, Rapoport JL, Delisi LE, Krebs MO, Joober R, Fathalli F, Mouaffak F, Haghighi AP, Néri C, et al. (2010) De novo mutations in the gene encoding the synaptic scaffolding protein SHANK3 in patients ascertained for schizophrenia. Proc Natl Acad Sci U S A 107:7863-7868.

Giese KP, Fedorov NB, Filipkowski RK, Silva AJ (1998) Autophosphorylation at Thr286 of the alpha calcium-calmodulin kinase II in LTP and learning. Science 279:870-873.

Grueter CE, Abiria SA, Wu Y, Anderson ME, Colbran RJ (2008) Differential regulated interactions of calcium/calmodulin-dependent protein kinase II with isoforms of voltage-gated calcium channel beta subunits. Biochemistry 47:1760-1767.

Gustin RM, Shonesy BC, Robinson SL, Rentz TJ, Baucum AJ 2nd, JalanSakrikar N, Winder DG, Stanwood GD, Colbran RJ (2011) Loss of Thr286 phosphorylation disrupts synaptic CaMKIIalpha targeting, NMDAR activity and behavior in pre-adolescent mice. Mol Cell Neurosci 47:286-292.

Halt AR, Dallapiazza RF, Zhou Y, Stein IS, Qian H, Juntti S, Wojcik S, Brose N, Silva AJ, Hell JW (2012) CaMKII binding to GluN2B is critical during memory consolidation. EMBO J 31:1203-1216.

Han K, Holder JL Jr, Schaaf CP, Lu H, Chen H, Kang H, Tang J, Wu Z, Hao S, Cheung SW, Yu P, Sun H, Breman AM, Patel A, Lu HC, Zoghbi HY (2013) SHANK3 overexpression causes manic-like behaviour with unique pharmacogenetic properties. Nature 503:72-77.

Harony-Nicolas H, De Rubeis S, Kolevzon A, Buxbaum JD (2015) Phelan McDermid syndrome: from genetic discoveries to animal models and treatment. J Child Neurol 30:1861-1870.

Hell JW (2014) CaMKII: claiming center stage in postsynaptic function and organization. Neuron 81:249-265.

Herbert MR (2011) SHANK3, the synapse, and autism. N Engl J Med 365:173-175

Hetzenauer A, Sinnegger-Brauns MJ, Striessnig J, Singewald N (2006) Brain activation pattern induced by stimulation of L-type $\mathrm{Ca}^{2+}$-channels: contribution of $\mathrm{Ca}(\mathrm{V}) 1.3$ and $\mathrm{Ca}(\mathrm{V}) 1.2$ isoforms. Neuroscience 139:1005-1015.
Jenkins MA, Christel CJ, Jiao Y, Abiria S, Kim KY, Usachev YM, Obermair GJ, Colbran RJ, Lee A (2010) $\mathrm{Ca}^{2+}$-dependent facilitation of Cav1.3 $\mathrm{Ca}^{2+}$ channels by densin and $\mathrm{Ca}^{2+} /$ calmodulin-dependent protein kinase II. J Neurosci 30:5125-5135.

Jiao Y, Jalan-Sakrikar N, Robison AJ, Baucum AJ 2nd, Bass MA, Colbran RJ (2011) Characterization of a central $\mathrm{Ca}^{2+} /$ calmodulin-dependent protein kinase IIalpha/beta binding domain in densin that selectively modulates glutamate receptor subunit phosphorylation. J Biol Chem 286:24806-24818.

Kandel ER (2012) The molecular biology of memory: cAMP, PKA, CRE, CREB-1, CREB-2, and CPEB. Mol Brain 5:14.

Kida S, Serita T (2014) Functional roles of CREB as a positive regulator in the formation and enhancement of memory. Brain Res Bull 105:17-24.

Küry S, van Woerden GM, Besnard T, Proietti Onori M, Latypova X, Towne MC, Cho MT, Prescott TE, Ploeg MA, Sanders S, Stessman HA, Pujol A, Distel B, Robak LA, Bernstein JA, Denommé-Pichon AS, Lesca G, Sellars EA, Berg J, Carré W, et al. (2017) De novo mutations in protein kinase genes CAMK2A and CAMK2B cause intellectual disability. Am J Hum Genet 101:768-788.

Lai Y, Nairn AC, Greengard P (1986) Autophosphorylation reversibly regulates the $\mathrm{Ca}^{2+}$ /calmodulin dependence of $\mathrm{Ca}^{2+} /$ calmodulin-dependent protein kinase II. Proc Natl Acad Sci U S A 83:4253-4257.

Leblond CS, Nava C, Polge A, Gauthier J, Huguet G, Lumbroso S, Giuliano F, Stordeur C, Depienne C, Mouzat K, Pinto D, Howe J, Lemière N, Durand CM, Guibert J, Ey E, Toro R, Peyre H, Mathieu A, Amsellem F, et al. (2014) Meta-analysis of SHANK mutations in autism spectrum disorders: a gradient of severity in cognitive impairments. PLoS Genet 10: e1004580.

Li B, Tadross MR, Tsien RW (2016) Sequential ionic and conformational signaling by calcium channels drives neuronal gene expression. Science (New York) 351:863-867.

Li Q, Lau A, Morris TJ, Guo L, Fordyce CB, Stanley EF (2004) A syntaxin 1, Galpha(o), and N-type calcium channel complex at a presynaptic nerve terminal: analysis by quantitative immunocolocalization. J Neurosci 24 : 4070-4081.

Limpitikul WB, Dick IE, Ben-Johny M, Yue DT (2016) An autismassociated mutation in $\mathrm{CaV} 1.3$ channels has opposing effects on voltageand $\mathrm{Ca}(2+)$-dependent regulation. Sci Rep 6:27235.

Lisman J, Yasuda R, Raghavachari S (2012) Mechanisms of CaMKII action in long-term potentiation. Nat Rev Neurosci 13:169-182.

Ma H, Groth RD, Cohen SM, Emery JF, Li B, Hoedt E, Zhang G, Neubert TA, Tsien RW (2014) $\gamma$ CaMKII shuttles $\mathrm{Ca}(2)(+) / \mathrm{CaM}$ to the nucleus to trigger CREB phosphorylation and gene expression. Cell 159:281-294.

Marks CR, Shonesy BC, Wang X, Stephenson JR, Niswender CM, Colbran RJ (2018) Activated CaMKIIalpha binds to the mGlu5 metabotropic glutamate receptor and modulates calcium mobilization. Mol Pharmacol 94:1352-1362.

McNeill RB, Colbran RJ (1995) Interaction of autophosphorylated $\mathrm{Ca}^{2+}$ calmodulin-dependent protein-kinase-II with neuronal cytoskeletal proteins: characterization of binding to a $190-\mathrm{kDa}$ postsynaptic density protein. J Biol Chem 270:10043-10049.

Miller SG, Kennedy MB (1986) Regulation of brain type II $\mathrm{Ca}^{2+}$ / calmodulin-dependent protein kinase by autophosphorylation: $\mathrm{Ca}^{2+}$ triggered molecular switch. Cell 44:861-870.

Miller SG, Patton BL, Kennedy MB (1988) Sequences of autophosphorylation sites in neuronal type II CaM kinase that control Ca2(+)-independent activity. Neuron 1:593-604.

Monteiro P, Feng G (2017) SHANK proteins: roles at the synapse and in autism spectrum disorder. Nat Rev Neurosci 18:147-157.

Moon AL, Haan N, Wilkinson LS, Thomas KL, Hall J (2018) CACNA1C: association with psychiatric disorders, behavior, and neurogenesis. Schizophr Bull 44:958-965.

Naisbitt S, Kim E, Tu JC, Xiao B, Sala C, Valtschanoff J, Weinberg RJ, Worley PF, Sheng M (1999) Shank, a novel family of postsynaptic density proteins that binds to the NMDA receptor/PSD-95/GKAP complex and cortactin. Neuron 23:569-582.

Nyegaard M, Demontis D, Foldager L, Hedemand A, Flint TJ, Sorensen KM, Andersen PS, Nordentoft M, Werge T, Pedersen CB, Hougaard DM, Mortensen PB, Mors O, Borglum AD (2010) CACNA1C (rs1006737) is associated with schizophrenia. Mol Psychiatry 15:119-121.

Orlandini M, Marconcini L, Ferruzzi R, Oliviero S (1996) Identification of a c-fos-induced gene that is related to the platelet-derived growth factor/ 
vascular endothelial growth factor family. Proc Natl Acad Sci U S A 93:11675-11680.

Peça J, Feliciano C, Ting JT, Wang W, Wells MF, Venkatraman TN, Lascola CD, Fu Z, Feng G (2011) Shank3 mutant mice display autistic-like behaviours and striatal dysfunction. Nature 472:437-442.

Pinggera A, Striessnig J (2016) Cav 1.3 (CACNA1D) L-type Ca(2+) channel dysfunction in CNS disorders. J Physiol 594:5839-5849.

Pinggera A, Lieb A, Benedetti B, Lampert M, Monteleone S, Liedl KR, Tuluc P, Striessnig J (2015) CACNA1D de novo mutations in autism spectrum disorders activate Cav1.3 L-type calcium channels. Biol Psychiatry $77: 816-822$.

Pinggera A, Mackenroth L, Rump A, Schallner J, Beleggia F, Wollnik B, Striessnig J (2017) New gain-of-function mutation shows CACNA1D as recurrently mutated gene in autism spectrum disorders and epilepsy. Hum Mol Genet 26:2923-2932.

Proietti Onori M, Koopal B, Everman DB, Worthington JD, Jones JR, Ploeg MA, Mientjes E, van Bon BW, Kleefstra T, Schulman H, Kushner SA, Küry S, Elgersma Y, van Woerden GM (2018) The intellectual disabilityassociated CAMK2G p.Arg292Pro mutation acts as a pathogenic gain-offunction. Hum Mutat 39:2008-2024.

Robison AJ, Bartlett RK, Bass MA, Colbran RJ (2005a) Differential modulation of $\mathrm{Ca}^{2+} /$ calmodulin-dependent protein kinase II activity by regulated interactions with $\mathrm{N}$-methyl-D-aspartate receptor NR2B subunits and alpha-actinin. J Biol Chem 280:39316-39323.

Robison AJ, Bass MA, Jiao Y, MacMillan LB, Carmody LC, Bartlett RK, Colbran RJ (2005b) Multivalent interactions of calcium/calmodulindependent protein kinase II with the postsynaptic density proteins NR2B, densin-180, and alpha-actinin-2. J Biol Chem 280:35329-35336.

Sala C, Futai K, Yamamoto K, Worley PF, Hayashi Y, Sheng M (2003) Inhibition of dendritic spine morphogenesis and synaptic transmission by activity-inducible protein Homer1a. J Neurosci 23:6327-6337.

Shanks NF, Maruo T, Farina AN, Ellisman MH, Nakagawa T (2010) Contribution of the global subunit structure and stargazin on the maturation of AMPA receptors. J Neurosci 30:2728-2740.

Shaywitz AJ, Greenberg ME (1999) CREB: a stimulus-induced transcription factor activated by a diverse array of extracellular signals. Annu Rev Biochem 68:821-861.

Shen K, Meyer T (1999) Dynamic control of CaMKII translocation and localization in hippocampal neurons by NMDA receptor stimulation. Science 284:162-166.

Shonesy BC, Jalan-Sakrikar N, Cavener VS, Colbran RJ (2014) CaMKII: a molecular substrate for synaptic plasticity and memory. Prog Mol Biol Transl Sci 122:61-87.

Soler J, Fananas L, Parellada M, Krebs MO, Rouleau GA, Fatjo-Vilas M (2018) Genetic variability in scaffolding proteins and risk for schizophrenia and autism-spectrum disorders: a systematic review. J Psychiatr Neurosci 43:170066.

Stephenson JR, Wang X, Perfitt TL, Parrish WP, Shonesy BC, Marks CR, Mortlock DP, Nakagawa T, Sutcliffe JS, Colbran RJ (2017) A novel human CAMK2A mutation disrupts dendritic morphology and synaptic transmission, and causes ASD-related behaviors. J Neurosci 37:2216-2233.

Strack S, Colbran RJ (1998) Autophosphorylation-dependent targeting of calcium/calmodulin-dependent protein kinase II by the NR2B subunit of theN-methyl- D-aspartate receptor. J Biol Chem 273:20689-20692.

Strack S, McNeill RB, Colbran RJ (2000) Mechanism and regulation of calcium/calmodulin-dependent protein kinase II targeting to the NR2B subunit of the N-methyl-D-aspartate receptor. J Biol Chem 275:23798 23806.

Trettel F, Rigamonti D, Hilditch-Maguire P, Wheeler VC, Sharp AH, Persichetti F, Cattaneo E, MacDonald ME (2000) Dominant phenotypes produced by the HD mutation in STHdh(Q111) striatal cells. Hum Mol Genet 9:2799-2809.

Tu JC, Xiao B, Naisbitt S, Yuan JP, Petralia RS, Brakeman P, Doan A, Aakalu VK, Lanahan AA, Sheng M, Worley PF (1999) Coupling of mGluR/ Homer and PSD-95 complexes by the shank family of postsynaptic density proteins. Neuron 23:583-592.

Uchino S, Waga C (2013) SHANK3 as an autism spectrum disorderassociated gene. Brain Dev 35:106-110.

Verpelli C, Dvoretskova E, Vicidomini C, Rossi F, Chiappalone M, Schoen M, Di Stefano B, Mantegazza R, Broccoli V, Böckers TM, Dityatev A, Sala C (2011) Importance of Shank3 protein in regulating metabotropic glutamate receptor 5 (mGluR5) expression and signaling at synapses. J Biol Chem 286:34839-34850.

Waga C, Asano H, Sanagi T, Suzuki E, Nakamura Y, Tsuchiya A, Itoh M, Goto Y, Kohsaka S, Uchino S (2014) Identification of two novel Shank3 transcripts in the developing mouse neocortex. J Neurochem 128:280-293.

Wang X, McCoy PA, Rodriguiz RM, Pan Y, Je HS, Roberts AC, Kim CJ, Berrios J, Colvin JS, Bousquet-Moore D, Lorenzo I, Wu G, Weinberg RJ, Ehlers MD, Philpot BD, Beaudet AL, Wetsel WC, Jiang YH (2011) Synaptic dysfunction and abnormal behaviors in mice lacking major isoforms of Shank3. Hum Mol Genet 20:3093-3108.

Wang X, Marks CR, Perfitt TL, Nakagawa T, Lee A, Jacobson DA, Colbran RJ (2017) A novel mechanism for $\mathrm{Ca}(2+) /$ calmodulin-dependent protein kinase II targeting to L-type $\mathrm{Ca}(2+)$ channels that initiates long-range signaling to the nucleus. J Biol Chem 292:17324-17336.

Weick JP, Groth RD, Isaksen AL, Mermelstein PG (2003) Interactions with PDZ proteins are required for L-type calcium channels to activate cAMP response element binding protein-dependent gene expression. J Neurosci 23:3446-3456.

Wheeler DG, Barrett CF, Groth RD, Safa P, Tsien RW (2008) CaMKII locally encodes L-type channel activity to signal to nuclear CREB in excitation-transcription coupling. J Cell Biol 183:849-863.

Wheeler DG, Groth RD, Ma H, Barrett CF, Owen SF, Safa P, Tsien RW (2012) $\mathrm{Ca}(\mathrm{V}) 1$ and $\mathrm{Ca}(\mathrm{V}) 2$ channels engage distinct modes of $\mathrm{Ca}(2+)$ signaling to control CREB-dependent gene expression. Cell 149:1112-1124.

Williams AJ, Umemori H (2014) The best-laid plans go oft awry: synaptogenic growth factor signaling in neuropsychiatric disease. Front Synaptic Neurosci 6:4.

Zhang H, Maximov A, Fu Y, Xu F, Tang TS, Tkatch T, Surmeier DJ, Bezprozvanny I (2005) Association of CaV1.3 L-type calcium channels with shank. J Neurosci 25:1037-1049.

Zhang H, Fu Y, Altier C, Platzer J, Surmeier DJ, Bezprozvanny I (2006) Ca1.2 and CaV1.3 neuronal L-type calcium channels: differential targeting and signaling to pCREB. Eur J Neurosci 23:2297-2310. 